\title{
SPECTRAL AND TIMING NATURE OF THE SYMBIOTIC X-RAY BINARY 4U 1954+319: THE SLOWEST ROTATING NEUTRON STAR IN AN X-RAY BINARY SYSTEM
}

\author{
Teruaki Enoto ${ }^{1,2}$, Makoto Sasano ${ }^{3}$, Shin'ya Yamada ${ }^{2}$, Toru Tamagawa ${ }^{2}$, Kazuo Makishima $^{2,3}$, Katja Pottschmidt $^{4,5}$, \\ Diana Marcu ${ }^{4,5}$, Robin H. D. CORBet ${ }^{1,5}$, Felix Fuerst $^{6}$, and Jörn Wilms ${ }^{7}$ \\ ${ }^{1}$ NASA Goddard Space Flight Center, Astrophysics Science Division, Code 662, Greenbelt, MD 20771, USA; teruaki.enoto@ nasa.gov \\ ${ }^{2}$ High Energy Astrophysics Laboratory, RIKEN Nishina Center, 2-1 Hirosawa, Wako, Saitama 351-0198, Japan \\ ${ }^{3}$ Department of Physics, University of Tokyo, 7-3-1 Hongo, Bunkyo-ku, Tokyo 113-0033, Japan \\ ${ }^{4}$ NASA Goddard Space Flight Center, Astrophysics Science Division, Code 661, Greenbelt, MD 20771, USA \\ ${ }^{5}$ CRESST and University of Maryland Baltimore County, Baltimore, MD 21250, USA \\ ${ }^{6}$ Cahill Center for Astronomy and Astrophysics, California Institute of Technology, Pasadena, CA 91125, USA \\ ${ }^{7}$ Dr. Remeis-Sternwarte and Erlangen Centre for Astroparticle Physics, Universität Erlangen-Nürnberg, Sternwartstr. 7, D-96049 Bamberg, Germany \\ Received 2014 January 9; accepted 2014 March 26; published 2014 April 24
}

\begin{abstract}
The symbiotic X-ray binary (SyXB) $4 \mathrm{U} 1954+319$ is a rare system hosting a peculiar neutron star (NS) and an M-type optical companion. Its $\sim 5.4 \mathrm{hr}$ NS spin period is the longest among all known accretion-powered pulsars and exhibited large $(\sim 7 \%)$ fluctuations over 8 yr. A spin trend transition was detected with Swift/BAT around an X-ray brightening in 2012. The source was in quiescent and bright states before and after this outburst based on 60 ks Suzaku observations in 2011 and 2012. The observed continuum is well described by a Comptonized model with the addition of a narrow $6.4 \mathrm{keV} \mathrm{Fe}-\mathrm{K} \alpha$ line during the outburst. Spectral similarities to slowly rotating pulsars in high-mass X-ray binaries, its high pulsed fraction $(\sim 60 \%-80 \%)$, and the location in the Corbet diagram favor high $B$-field ( $\gtrsim 10^{12} \mathrm{G}$ ) over a weak field as in low-mass X-ray binaries. The observed low X-ray luminosity $\left(10^{33}-10^{35} \mathrm{erg} \mathrm{s}^{-1}\right)$, probable wide orbit, and a slow stellar wind of this SyXB make quasi-spherical accretion in the subsonic settling regime a plausible model. Assuming a $\sim 10^{13} \mathrm{G} \mathrm{NS}$, this scheme can explain the $\sim 5.4 \mathrm{hr}$ equilibrium rotation without employing the magnetar-like field $\left(\sim 10^{16} \mathrm{G}\right)$ required in the disk accretion case. The timescales of multiple irregular flares $(\sim 50 \mathrm{~s})$ can also be attributed to the free-fall time from the Alfvén shell for a $\sim 10^{13} \mathrm{G}$ field. A physical interpretation of SyXBs beyond the canonical binary classifications is discussed.
\end{abstract}

Key words: accretion, accretion disks - binaries: symbiotic - magnetic fields - stars: individual (3A 1954+319, 4U 1954+319) - stars: neutron - X-rays : binaries

Online-only material: color figures

\section{INTRODUCTION}

Symbiotic X-ray binaries (SyXBs) are a growing new subclass of X-ray binaries which consist of an X-ray bright neutron star (NS) and an M-type giant primary star. Originally SyXBs were seen as a subclass of low-mass X-ray binaries (LMXBs), where accretion typically is from a K-type optical counterpart with a mass $M_{\mathrm{c}} \lesssim 1 M_{\odot}$. It took more than $30 \mathrm{yr}$, however, to recognize that their properties are different from LMXBs and also from those of high-mass binaries (HXMBs), i.e., systems where the mass donor is an $\mathrm{O}$ - or B-type giant with $M_{\mathrm{c}} \gtrsim 10 M_{\odot}$. SyXBs are now considered as a separate subclass of X-ray binaries. They are named in analogy to symbiotic binaries, which consist of a white dwarf and an M-giant companion, but their nature has not yet been understood.

Some SyXBs were known as famous X-ray sources since the early X-ray observations: GX $1+4$ (Davidsen et al. 1977; Makishima et al. 1988; Chakrabarty \& Roche 1997), 4U 1700+24 (Garcia et al. 1983; Masetti et al. 2002), and Sct X-1 (Kaplan et al. 2007). With the discovery of new sources such as IGR J16194-2810, there are now nearly five SyXBs and some candidates in our Galaxy; e.g., five objects out of ten candidates (Table 1 in Lü et al. 2012) have been confirmed as SyXBs, while three were ruled out (see, e.g., Masetti et al. 2012). Observational features of the SyXB class are (1) quite long orbital periods, ${ }^{8}$ e.g., 1161 days in GX 1+4; (2) long NS

\footnotetext{
8 For many SyXB, the orbital period has not yet been known nor confirmed in long-term X-ray studies (e.g., Corbet et al. 2008).
}

spin periods over $\sim 110-18300 \mathrm{~s}$ as shown in Figure 1; and (3) high X-ray variability ranging from short to long timescales $(\sim 1 \mathrm{~s}$ to up to a year).

SyXB X-ray spectra are in general well described by an absorbed power law with a photon index $\Gamma \sim 0.5-2.0$ and a high energy roll-over, which is often modified in the soft $\mathrm{X}$-rays by strong absorption. This spectrum resembles that of X-ray pulsars in HMXBs, and thus, SyXBs have been interpreted in the literature as NSs with $\sim 10^{12} \mathrm{G}$ field in HMXBs. As another interpretation, following the classical LMXB classification based on optical companions, SyXBs were also interpreted as NSs in the low-luminosity low/hard state of LMXB as having weak magnetic field ( $B$-field), e.g., $\lesssim 10^{10} \mathrm{G}$ (Nagae et al. 2008; Kitamura et al. 2014). In any of these cases, the NSs in SyXB are expected to have $B$-fields strengths of around $10^{12} \mathrm{G}$ or much lower (e.g., $\lesssim 10^{10} \mathrm{G}$ ).

The canonical $B$-fields implied by the spectral analogy with HMXBs and LMXBs, however, are in contradiction to the fields implied by their timing behavior. The slow NS pulsations in SyXBs are sometimes in spin equilibrium or show a large spinup rate (Makishima et al. 1988; González-Galán et al. 2012). If interpreted in terms of standard disk accretion torque theory (Ghosh \& Lamb 1979), the slow rotation and torque reversals imply extraordinary strong dipole fields $\left(\sim 10^{14-16} \mathrm{G}\right)$, which are close to or exceed the quantum critical field, $B_{\mathrm{cr}}=m_{\mathrm{e}}^{2} c^{3} /(e \hbar)=$ $4.4 \times 10^{13} \mathrm{G}$. Such a magnetar scenario for binary systems has been proposed, e.g., to explain the $1.6 \mathrm{hr}$ pulsation and the high spin-up rate of the SyXB IGR J16358-4726 (Patel et al. 


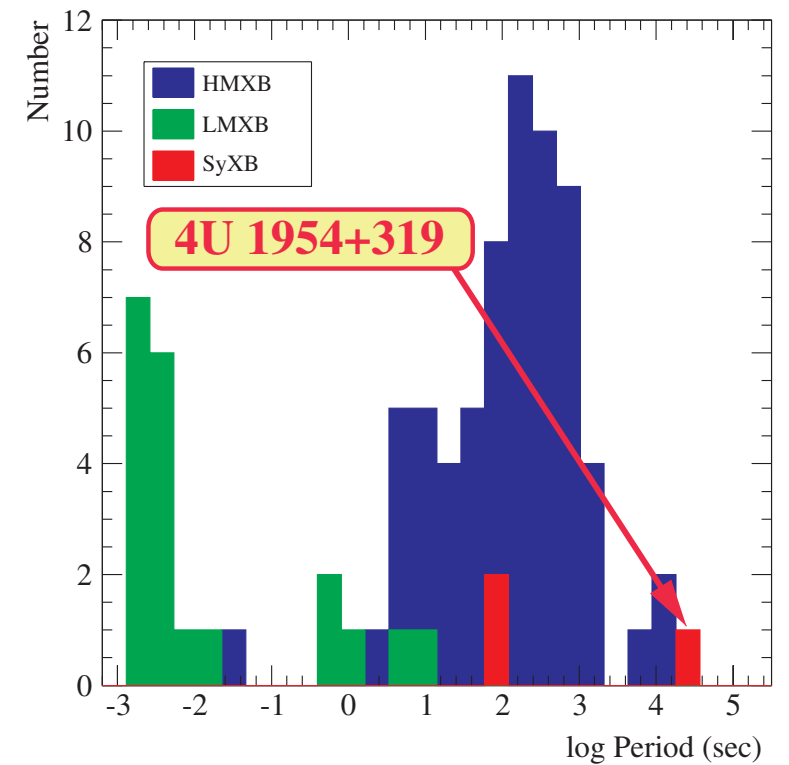

Figure 1. Distribution of NS rotation periods in HMXBs (Liu et al. 2006), LMXBs (Liu et al. 2007), and confirmed SyXBs. Measured rotation periods of SyXBs are $\sim 18,300 \mathrm{~s}(4 \mathrm{U} 1954+319), \sim 110 \mathrm{~s}(\mathrm{Sct} X-1)$, and $\sim 150 \mathrm{~s}(\mathrm{GX} 1+4)$. The $4 \mathrm{U} 1954+319$ is in the HMXB range.

(A color version of this figure is available in the online journal.)

2007). Similar scenarios have also been discussed for slowly rotating X-ray pulsars in HMXBs such as 4U 0114+65 (Li \& van den Heuvel 1999) or 4U 2206+54 (Reig et al. 2012), highly variable supergiant fast X-ray transients (Bozzo et al. 2008), and some slowly rotating NSs in the Small Magellanic Cloud (Ho et al. 2014; Klus et al. 2014). Except for the timing properties, however, no direct evidence for ultra-strong $B$-fields has been found so far (e.g., Ikhsanov 2007).

As shown by Shakura et al. (2012) and Postnov et al. (2010, 2012), the extremely strong fields can be avoided when one assumes that the accretion does not happen through an accretion disk but via quasi-spherical accretion. In these models, matter is gravitationally captured, e.g., from the donor's stellar wind. For low mass accretion rates, $\dot{M} \lesssim 4 \times 10^{16} \mathrm{~g} \mathrm{~s}^{-1}$, gravitationally captured matter subsonically accretes onto the NS forming an extended, quasi-spherical shell around the magnetosphere, where large scale convective motions and subsonic turbulence lead to accretion onto the NS. Contrary to the canonical highluminosity Bondi-Hoyle accretion, where matter supersonically accretes and the sign of angular momentum (prograde or retrograde) determines the spin behavior (Perna et al. 2006), for quasi-spherical accretion the spin-up/spin-down is determined by the specific angular momentum of matter at the magnetospheric boundary and the angular velocity of the NS.

In this paper, we discuss Suzaku observations of the SyXB 4U 1954+319 hosting the slowest rotating NS in an X-ray binary system. This source was originally discovered by Uhuru (Forman et al. 1978) and observed in the 1980s with Ariel (Warwick et al. 1981), EXOSAT, and Ginga (Tweedy et al. 1989). These observations suggested that the source was a wind accreting HMXB with a very inhomogeneous wind (Tweedy et al. 1989), although no optical counterpart was known at that time. The source had virtually been forgotten until, after $20 \mathrm{yr}$ of no observations, Chandra's localization of the source position allowed the optical identification of the donor, which was found to be an M4-5 III star $\left(M_{\mathrm{c}} \sim 1.2 M_{\odot}\right)$ at a distance of $1.7 \mathrm{kpc}$
(Masetti et al. 2006b). Therefore, 4U 1954+319 was re-classified as a SyXB.

Further progress in our understanding of the characteristics came with the discovery of a $\sim 5 \mathrm{hr}$ periodicity in Swift/BAT monitoring data by Corbet et al. (2006, 2008). This period is strongly variable (Marcu et al. 2011). So far, no orbital period has been discovered, although Mattana et al. (2006) argue that the lower limit of the orbital period is $\sim 400$ days. These parameters are incompatible with typical white dwarf systems, such that the compact object in 4U 1954+319 is likely a NS. As shown in Figure 1, the extremely long spin period makes 4U 1954+319 the slowest rotating accretion-powered NS system known to date ${ }^{9}$ and a prominent example for understanding the SyXB class.

Currently, no consensus has yet been obtained on the accretion models for SyXBs, or the $B$-field of their NSs. Revisiting and developing the quasi-spherical model further requires the spectral and timing investigations of SyXBs. In this paper, we report on the broadband timing and spectral properties of $4 \mathrm{U}$ 1954+319 and discuss a possible accretion model beyond the conventional HMXB and LMXB classification.

\section{OBSERVATION AND DATA REDUCTION}

\subsection{Suzaku Observations}

There are two pointed Suzaku (Mitsuda et al. 2007) observations of $4 \mathrm{U} 1954+319$. The first one, in AO6, was conducted on 2011 October 23-24 for a gross on-source time of $117 \mathrm{ks}$. Nearly 1 yr later, on 2012 October 5, MAXI monitoring detected a brightening of this source, ${ }^{10}$ with the X-ray intensity reaching $\sim 15 \mathrm{mCrab}$ in the $3-10 \mathrm{keV}$ band. About 27 days later, on 2012 November $1 / 2$, a follow-up target of opportunity (ToO) observation was performed with Suzaku for a gross on-source time of 120 ks. Table 1 summarizes the two Suzaku observations.

The X-ray Imaging Spectrometer (XIS; Koyama et al. 2007) on board Suzaku, coupled to the X-ray Telescopes (XRT; Serlemitsos et al. 2007), consists of two front-illuminated (FI) CCD detectors, XIS0 and XIS3, and one back-illuminated sensor (XIS1). The CCDs are sensitive in the $0.2-12 \mathrm{keV}$ range and, when operated in the full window mode, read out every $8 \mathrm{~s}$. We also used data from the hard X-ray detector (HXD; Takahashi et al. 2007), which consists of Silicon PIN diodes (HXD-PIN) and GSO scintillators (HXD-GSO) and covers the 10-70 keV and 50-600 keV bands, respectively. The HXD was operated in the nominal mode during both observations with $61 \mu$ s time resolution. The bright $\mathrm{X}$-ray source Cygnus $\mathrm{X}$ 1 , which is only 3.15 from $4 \mathrm{U} 1954+319$, is still outside the HXD-PIN's tightly collimated field of view (FOV; $34^{\prime} \times 34^{\prime}$ FWHM) but is within the FOV of the HXD-GSO $\left(4^{\circ} \times 4^{\circ}\right)$. Thus, we did not use the HXD-GSO data in the following analyses due to a possible contamination from Cyg X-1. Based on the INTEGRAL source catalog, there is no other strong contamination source within the HXD-PIN FOV.

\subsection{Data Processing}

We analyzed the XIS and HXD data sets as obtained from the standard pipeline processing, revision 2.7 and 2.8 for the

\footnotetext{
9 A long periodicity, $P \sim 6.67 \mathrm{hr}$, was also detected from the central object 1E161348-5055 in the young shell-type supernova remnant RCW 103. This object, however, has not been confirmed as an accretion-powered pulsar. 10 Trigger ID 6206800002:

http://maxi.riken.jp/pipermail/x-ray-star/2012-October/000187.html.
} 
Table 1

Log of Suzaku Observations of 4U 1954+319

\begin{tabular}{|c|c|c|}
\hline & $\begin{array}{l}2011 \\
\text { AO6 }\end{array}$ & $\begin{array}{c}2012 \\
\text { AO7 ToO }\end{array}$ \\
\hline Observation start & Oct. 23 08:05 & Nov. 1 02:39 \\
\hline Start MJD & 55857.337 & 56232.110 \\
\hline Observation end & Oct. 24 17:17 & Nov. 2 12:02 \\
\hline End MJD & 55858.720 & 56233.501 \\
\hline Nominal position & XIS & XIS \\
\hline ObsID & 406046010 & 907005010 \\
\hline XIS signal rate $\left(\mathrm{cnt} \mathrm{s}^{-1}\right)$ & $0.18 / 0.18$ & $5.1 / 4.5$ \\
\hline XIS exposure (ks) & 60.2 & 60.8 \\
\hline PIN signal rate $\left(\mathrm{cnt} \mathrm{s}^{-1}\right)$ & $\ldots$ & 0.70 \\
\hline PIN exposure (ks) & 53.7 & 50.5 \\
\hline $\mathrm{X}$-ray Intensity & $0.28 \mathrm{mCrab}$ & $9.5 \mathrm{mCrab}$ \\
\hline$F_{\mathrm{X}}\left(10^{-12} \mathrm{erg} \mathrm{s}^{-1} \mathrm{~cm}^{-2}\right) 2-10 \mathrm{keV}$ & $5.36 \pm 0.03$ & $190.0_{-0.5}^{+0.2}$ \\
\hline$F_{\mathrm{X}}\left(10^{-12} \mathrm{erg} \mathrm{s}^{-1} \mathrm{~cm}^{-2}\right) 1-70 \mathrm{keV}$ & $6.28_{-0.52}^{+2.69}$ & $600.4_{-4.1}^{+3.1}$ \\
\hline$L_{\mathrm{x}}\left(\mathrm{erg} \mathrm{s}^{-1}\right) 1-70 \mathrm{keV}$ & $2.2 \times 10^{33}$ & $2.1 \times 10^{35}$ \\
\hline
\end{tabular}

Notes. The 0.5-10 keV XIS and 12-70 keV HXD-PIN signal rates are averaged during the observation. The XIS rates are shown for the XIS-FI and -BI CCD imagers. The source and background regions are stated in Section 2.2. The X-ray intensity is evaluated in the $2-10 \mathrm{keV}$ band. $F_{\mathrm{X}}$ and $L_{\mathrm{X}}$ are evaluated using the comptt model.

2011 and 2012 data sets, respectively. All data analyses were performed using HEADAS version 6.11 or later. Following the standard Suzaku screening criteria, X-ray events were discarded if one of the following conditions held true: (1) they were recorded during the South Atlantic Anomaly, TSAA $\leqslant 436 \mathrm{~s}$ and TSAA $\leqslant 500$ s for the XIS and the HXD, respectively, and later than $180 \mathrm{~s}$ before the next passage for the HXD; (2) while the satellite was in regions of low geomagnetic cutoff rigidity ( $\leqslant 6 \mathrm{GV})$; (3) while the target was closer than $5^{\circ}$ to Earth's rim; (4) if the instantaneous pointing direction deviated more than 1.5 from the mean; or (5) during periods of telemetry saturation. After screening, the net exposures for the 2011 observation were $60.2 \mathrm{ks}$ (XIS) and $53.7 \mathrm{ks}$ (HXD), while those in 2012 were $60.8 \mathrm{ks}$ (XIS) and $50.5 \mathrm{ks}$ (HXD), respectively.

On-source XIS events were extracted from a region of 2.5 radius centered on the source. Background events were derived from an annulus 4.0-7.5 around the source. From the HXD-PIN data, we subtracted the non X-ray Background (NXB) created with the LCFITD method (Fukazawa et al. 2009) and filtered following the same screening criteria as those used for the on-source data. The cosmic X-ray Background (CXB) was modeled as described by Enoto et al. (2010a) with the same spectral shape as given by Moretti et al. (2009). In the following analyses, unless otherwise specified, all uncertainties are given at the $68 \%(1 \sigma)$ confidence level.

\subsection{Reduction of the X-Ray Spectra}

For the spectral analysis, we summed the two XIS-FI sensors and utilized XIS data from the $0.8-10 \mathrm{keV}$ band and the HXD-PIN data from the $15-70 \mathrm{keV}$ band. The 1.7-1.9 keV XIS data are discarded due to calibration uncertainties. Uncertainties in the flux normalization of the instruments are taken into account with a multiplicative constant. The cross-normalization factor of HXD-PIN relative to XIS-FI was fixed at 1.171 based on the XIS-nominal Crab Nebula calibration, ${ }^{11}$ while

\footnotetext{
11 http://www.astro.isas.jaxa.jp/suzaku/doc/suzakumemo/ suzakumemo-2008-06.pdf
}

that of XIS-FI and XIS-BI was allowed to differ by up to $1 \%$. A $0.5 \%$ systematic uncertainty was added in quadrature to the 2012 XIS spectral bins in order to absorb instrumental calibration uncertainties. The response matrix files and auxiliary response file for the XIS were produced using the FTOOLs xisrmfgen and xissimarfgen (Ishisaki et al. 2007), while standard detector response files (epoch 11) were used for the HXD-PIN.

\section{SUZAKU OBSERVATIONS OF 4U 1954+319}

\subsection{Long-term Variability}

Figure 2 shows the long-term variability of the X-ray intensity of $4 \mathrm{U} 1954+319$, covering $\sim 17 \mathrm{yr}$ since 1994 . X-ray count rates were re-binned to 6 and 12 days resolution and converted into the mCrab unit using conversion factors provided by the RXTE/ All-Sky Monitor (ASM) and Swift/Burst Alert Telescope (BAT) teams. ${ }^{12}$ As shown in the 20 day running average of the fluxes (Figure 2, blue curve), the X-ray intensity of 4U 1954+319 is highly variable on a timescale of years, with fluxes changing from a few mCrab to at least $20 \mathrm{mCrab}$ or more. In addition, faster variability on timescales of weeks or days is also seen with irregular flares.

Figure 2 also shows the spin history of $4 \mathrm{U} 1954+319$ as measured with Swift/BAT. We determined the pulse periods using standard epoch folding (Leahy et al. 1983) and using pulse profiles with 19 phase bins. Spin values are shown with filled circles if pulses are detected with $\chi_{\text {red }}^{2}>3$. The measured period history is consistent with previous work covering data taken before 2010 (Marcu et al. 2011). The present work extends the analysis until the end of 2012 and shows the detection of a new change from spin-down to spin-up which occurred around 2012 March (MJD 56000), when the average X-ray intensity increased to $\sim 25 \mathrm{mCrab}$. The analysis shows that the first and second Suzaku observations were taken during the spindown and spin-up phases, respectively. The former observation was almost in quiescence ( $\sim 0.3 \mathrm{mCrab})$, before the outburst, while the latter was during a brighter phase after the flaring ( $\sim 10 \mathrm{mCrab})$ and the spin-transition.

\subsection{Timing Analysis}

Figure 3 shows the XIS and HXD-PIN light curves of 4U $1954+319$ from the two observations. Similar to the behavior observed on long timescales (Figure 2), the X-ray light curve is highly variable also on short timescales. Figure 4 further illustrates the enlarged light curve. The X-ray fluctuation was moderate in 2011, while spiky flare events were seen in 2012 with $\lesssim 100 \mathrm{~s}$ scale, showing a rapid flux change by an order of magnitude or more. During the 2012 outburst (Figure 4), the spiky flares become enhanced in the latter part of observation. An increase of the short-term fluctuation is also seen in the power spectral density (PSD) in Figure 5. The PSD continuum can be described by a power-law components with best-fit slopes of -0.77 in 2011 and -1.66 in 2012 . There are no signatures of clear short-term periods or quasi periodic oscillations. No low-frequency roll over was detected, either.

\footnotetext{
12 The long-term RXTE/ASM monitoring data are provided by the RXTE/ASM team (http://xte.mit.edu), while the Swift/BAT transient monitor results provided by the Swift/BAT team

(http://heasarc.gsfc.nasa.gov/docs/swift/results/transients/). The reported conversion factors, assuming a Crab-like spectrum, are

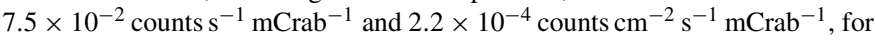
ASM and BAT, respectively.
} 


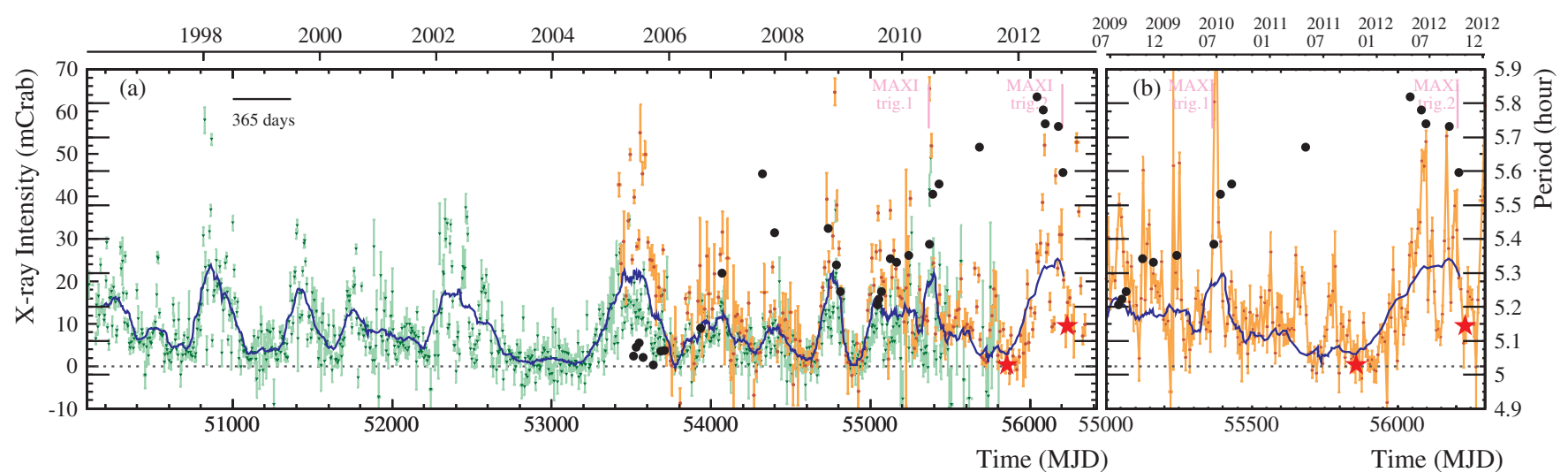

Figure 2. Long-term light curve of $4 \mathrm{U} 1954+319$. (a) 2-10 keV RXTE/ASM (triangle, green) and 15-50 keV Swift/BAT (filled circle, orange) light curves of $4 \mathrm{U}$ 1954+319 since 1994. The solid blue curve represents a running average of the X-ray intensity with a window size of 20 days. The detected pulse period history by the Swift/BAT is overlaid as black filled circles (right hand axis). Two MAXI detections of the flaring activity of this source are also shown: MAXI trig 1 on 2012 June 29 (Sugizaki et al. 2010) and trig 2 on 2012 October 5 (see the text). The two Suzaku observations are indicated as red star symbols. (b) Enlarged version of the light curves around the two Suzaku observations, showing the 7 days averaged X-ray intensity from Swift/BAT (orange).

(A color version of this figure is available in the online journal.)
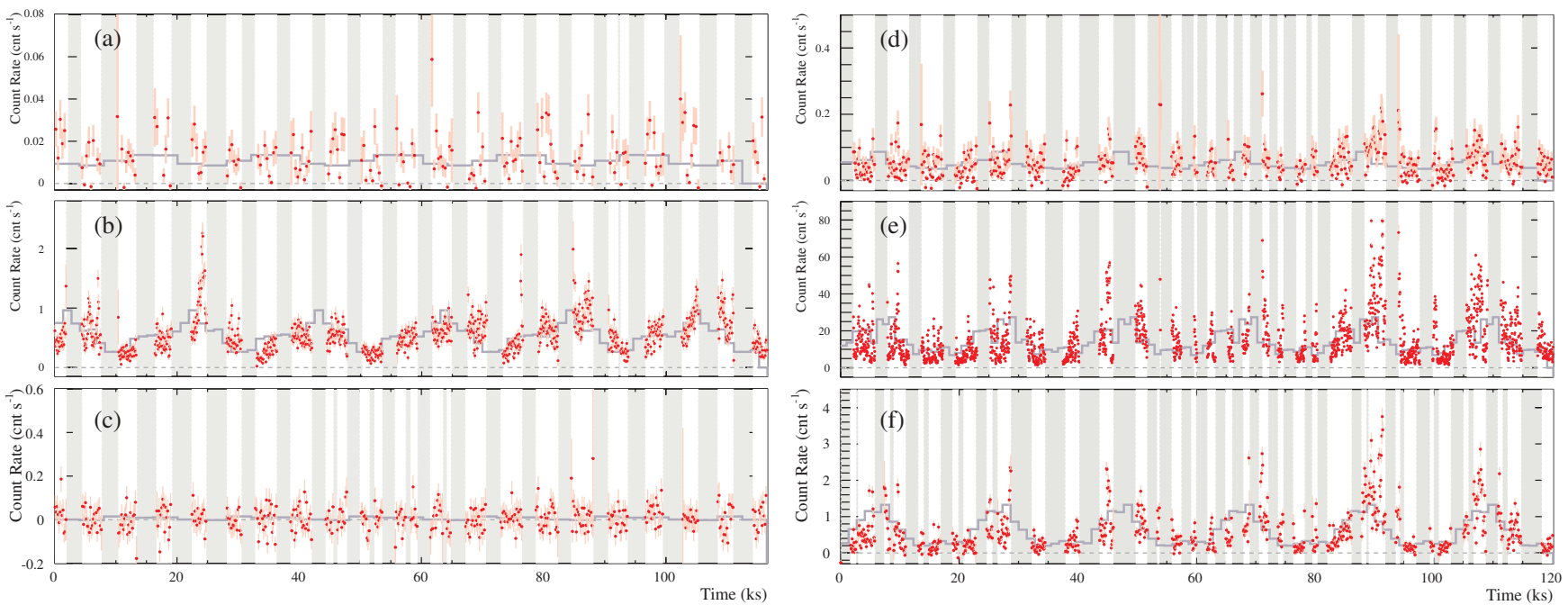

Figure 3. Background-subtracted light curves of 4 U 1954+319 obtained with Suzaku in 2011 October (left) and 2012 November (right). Gray strips represent the non good time intervals (non-GTIs) during the observation. From top to bottom, the panels show the 0.5-1.0 keV XIS (binning $360 \mathrm{~s} \mathrm{(2011)} \mathrm{and} \mathrm{120} \mathrm{s} \mathrm{(2012)),} \mathrm{1-10} \mathrm{keV}$ XIS (64 s and $32 \mathrm{~s}$ ), and 15-70 keV HXD-PIN (160 s and $80 \mathrm{~s}$ ) count rates. The XIS background was subtracted as described in Section 2.2, while the simulated NXB $\left(\sim 0.28\right.$ count s$\left.^{-1}\right)$ and CXB $\left(\sim 0.02\right.$ counts s$\left.{ }^{-1}\right)$ were subtracted from the dead-time-corrected HXD-PIN data. Estimated pulse shapes are overlaid in each panel, using the Swift/BAT period described in Section 3.2.

(A color version of this figure is available in the online journal.)

Epoch folding of the 2012 XIS and HXD data yielded a spin period of $5.68 \pm 0.28 \mathrm{hr}$. However, the period determination with Suzaku is not accurate enough, due to the limited exposure which corresponds to only $\sim 6$ cycles of the NS rotation. Thus, we searched the Swift/BAT monitoring data (Section 3.1) for the spin period using intervals of \pm 60 days and \pm 400 days around the Suzaku pointings. The pulse periods derived from these data are $P=5.70 \pm 0.01 \mathrm{hr}\left(\chi^{2} / v=104.7 / 14=7.5\right)$ and $P=5.76 \pm 0.01 \mathrm{hr}\left(\chi^{2} / \nu=72.3 / 14=5.2\right)$, in 2011 and 2012 , respectively, where 15 phase bins were used for the epoch folding. Average pulse profiles, obtained at these periods, are superposed in Figure 3 on the Suzaku light curves. Even though sporadic flares are rather strong, we can trace individual pulses in most cases.

Figure 6 shows the pulse profiles of the two Suzaku observations. The pulse profile in 2012 is approximately sinusoidal below $1 \mathrm{keV}$, with a single peak at phase $\sim 0.4$. A second peak at phase $\sim 0.2$ appears at higher energies. Figure 7 shows the energy dependence of the pulsed fraction (PF) of 4U 1954+319, defined as $\mathrm{PF}=\left(R_{\max }-R_{\min }\right) /\left(R_{\max }+R_{\min }\right)$, where $R_{\max }$ and $R_{\min }$ are the maximum and minimum of the pulse profile. The fraction is $60 \%-80 \%$ in the $0.5-30 \mathrm{keV}$ band and increases with energy. This is a typical trend often seen in X-ray pulsars in HMXBs (Lutovinov \& Tsygankov 2008).

\subsection{X-Ray Spectrum During the Flare in 2012}

Figure 8(a) shows Suzaku spectra of 4U 1954+319 obtained on the two occasions. We superpose the modeled HXD-PIN NXB and CXB, together with the typical $1 \%$ systematic uncertainty of the NXB. The HXD-PIN detection is marginal in 2011. The ratio between the two spectra is shown in Figure 8(b). In 2012 , 4U $1954+319$ was brighter by nearly $1-2$ orders of magnitude and exhibited harder spectra.

We now turn to modeling the X-ray spectrum in Figure 9, and summarize the best-fit parameters in Table 2. Fitting the data with a single, absorbed power law leaves a soft excess below $2 \mathrm{keV}$, an iron line at $\sim 6.4 \mathrm{keV}$, and a spectral roll over (Figure 9(a)). Here we use the latest interstellar absorption 


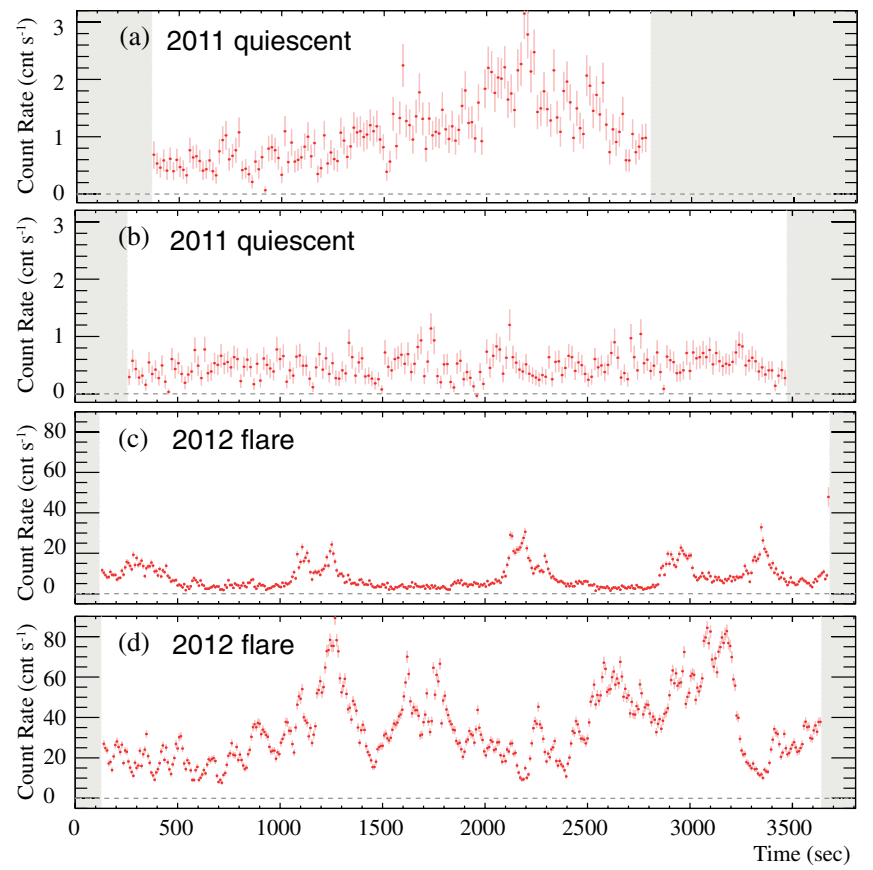

Figure 4. XIS 1-10 keV light curves during the 2011 (a, b) and the 2012 observation (c, d). Data are binned to $8 \mathrm{~s}$ resolution. Time durations are (a) $22.0-25.8 \mathrm{ks}$, (b) $55.8-59.6 \mathrm{ks}$, (c) $13.6-17.4 \mathrm{ks}$, and (d) $88.2-92.0 \mathrm{ks}$ in Figure 3.

(A color version of this figure is available in the online journal.)

model tbnew (Wilms et al. 2000) with an improved abundance wilm and cross section tables vern (Verner et al. 1996). Due to higher data quality compared to earlier INTEGRAL measurements, the broken power-law model used by Fürst et al. (2011b) and Marcu et al. (2011) was not successful $\left(\chi_{v}^{2}=2373.6 / 697=3.40\right)$, either. The exponentially cutoff power law with an additional soft blackbody and an Fe-line, improved the fit to $\chi^{2} / v=971.5 / 696=1.40$ (Figure 9(b)) with a photon index of $\Gamma=1.21$, a cutoff energy $E_{\text {cut }}=20 \mathrm{keV}$, and a blackbody temperature of $k T_{\mathrm{bb}}=0.13 \mathrm{keV}$. The spectral parameters are similar to those found by Mattana et al. (2006) by applying the same model to the BeppoSAX data, although our fit is not satisfactory.

As alternative representations of the roll-over of the continuum, we employed two empirical models: (1) a high energy cutoff model of the form (in XSPEC notation)

$$
\text { phabs } \times \text { (bbodyrad+gauss }+ \text { power law } \times \text { highecut }) \text {, }
$$

where

$$
\text { highecut }= \begin{cases}1 & \text { for } E<E_{\mathrm{c}} \\ \exp \left[\left(E_{\mathrm{c}}-E\right) / E_{\mathrm{f}}\right] & \text { for } E \geqslant E_{\mathrm{c}}\end{cases}
$$

with the cutoff energy $E_{\mathrm{c}} \sim 4.2 \mathrm{keV}$ and $e$-folding energy $E_{\mathrm{f}} \sim 21.0 \mathrm{keV}$. We also modeled the data using the (2) a negative and positive power law with an exponential (NPEX),

$$
\text { phabs } \times(\text { bbodyrad + gauss + cutoffpl + cutoffpl }),
$$

which is often utilized to represent broadband continuum from the accretion column of highly magnetized NSs (Mihara 1995; Makishima et al. 1999; Enoto et al. 2008). The cutoff energy and the photon index of the NPEX continuum were obtained as $6.6 \mathrm{keV}$ and 0.77 , respectively, and the blackbody temperature as $0.14 \mathrm{keV}$. As shown in Figures 9(c), (d), and Table 2, both

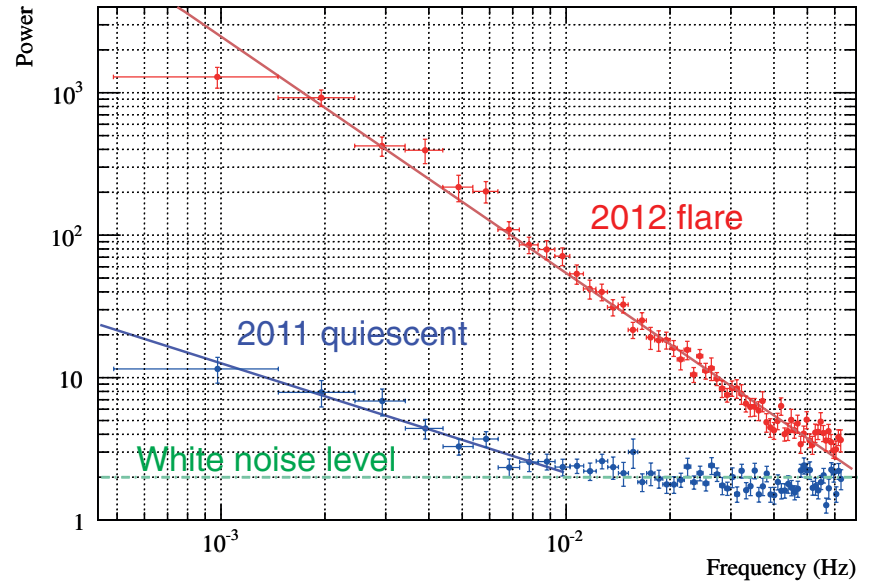

Figure 5. Power spectrum densities (PSDs) of the $0.8-10 \mathrm{keV}$ XIS data in 2011 and 2012 observations as calculated with the FTOOL powspec using the background-subtracted $8 \mathrm{~s}$ binned light curve. The PSDs are normalized such that the white noise level corresponds to 2 . The best-fit power-law models are shown with slopes of -0.77 and -1.66 in 2011 and 2012, respectively, when fitted above the white noise level.

(A color version of this figure is available in the online journal.)

models reproduced the broadband spectra quite well $\left(\chi_{v}^{2} \sim 1.2\right)$. If we exclude the soft blackbody, $\chi^{2}$ becomes worse $\left(\chi_{v}^{2}>3.5\right)$. Replacing the blackbody component with a diffuse plasma emission model (apec ${ }^{13}$; Smith et al. 2001), did not give acceptable fits $\left(\chi_{v}^{2}>1.9\right)$.

Finally, as a more physically based interpretation, we also tried an optically thick Comptonization model, compTT (Titarchuk \& Lyubarskij 1995). This gave an acceptable fit, $\chi_{v}^{2}=780.8 / 697=1.12$, with a null hypothesis probability of 0.02 (Figure 9(e)). We therefore adopt this model as a canonical continuum model of $4 \mathrm{U} 1954+319$. The derived best-fit parameters are a soft photon (Wien) temperature of $k T_{0} \sim 1 \mathrm{keV}$, plasma temperature $k T_{\mathrm{e}} \sim 8 \mathrm{keV}$, and an optical depth of $\tau \sim 9.0$. These derived $k T_{0}$ and $k T_{\mathrm{e}}$ values are within the typical range of the comptt model of this source, $k T_{0} \sim 0.6-1.3 \mathrm{keV}$ and $k T_{\mathrm{e}} \sim 3.0-13 \mathrm{keV}$, by the multi X-ray satellite studies (Masetti et al. 2007b) and by the INTEGRAL observation (Marcu et al. 2011). Combining the previous two successful models, the broadband spectrum is explained by the Comptonized model. Figure 10 gives a $v F_{v}$ representation of the data and the model. The 2-10 keV and 1-70 keV absorbed X-ray fluxes are (1.90 \pm $0.01) \times 10^{-10}$ and $(6.0 \pm 0.1) \times 10^{-10} \mathrm{erg} \mathrm{s}^{-1} \mathrm{~cm}^{-2}$, translating to absorption-corrected X-ray fluxes of $(2.03 \pm 0.01) \times 10^{-10}$ and $(6.3 \pm 0.1) \times 10^{-10} \mathrm{erg} \mathrm{s}^{-1} \mathrm{~cm}^{-2}$, respectively.

\subsection{Iron Line and Search for CRSF}

The $6.4 \mathrm{keV}$ neutral $\mathrm{Fe}-\mathrm{K} \alpha$ line emission is obvious in Figure 9(a). In the compTT continuum fits, its center energy was found at $6.375_{-0.008}^{+0.010} \mathrm{keV}$ with an equivalent width (EW) of $\mathrm{EW}=28.6 \pm 0.3 \mathrm{eV}$ and a total line flux of $I_{\mathrm{Fe}}=$ $7.5_{-0.7}^{+0.8} \times 10^{-5}$ photon $\mathrm{cm}^{-2} \mathrm{~s}^{-1}$. The line is narrow with a width $\sigma<50 \mathrm{eV}(1 \sigma)$, corresponding to a Doppler width $v_{\infty} \lesssim 2400 \mathrm{~km} \mathrm{~s}^{-1}$.

Detailed inspection of the spectrum indicates a shallow dent at $\sim 7.1 \mathrm{keV}$, and if we let the iron abundance free, the data favor the 2.4 times larger iron abundance relative to the default improving the fit by $\Delta \chi^{2}=15.3$ for $\Delta v=-1(F$-test probability 13 Astrophysical Plasma Emission Code, a collisonally ionzied diffuse gas
model http://atomdb.org/. 


\section{Suzaku 2011}
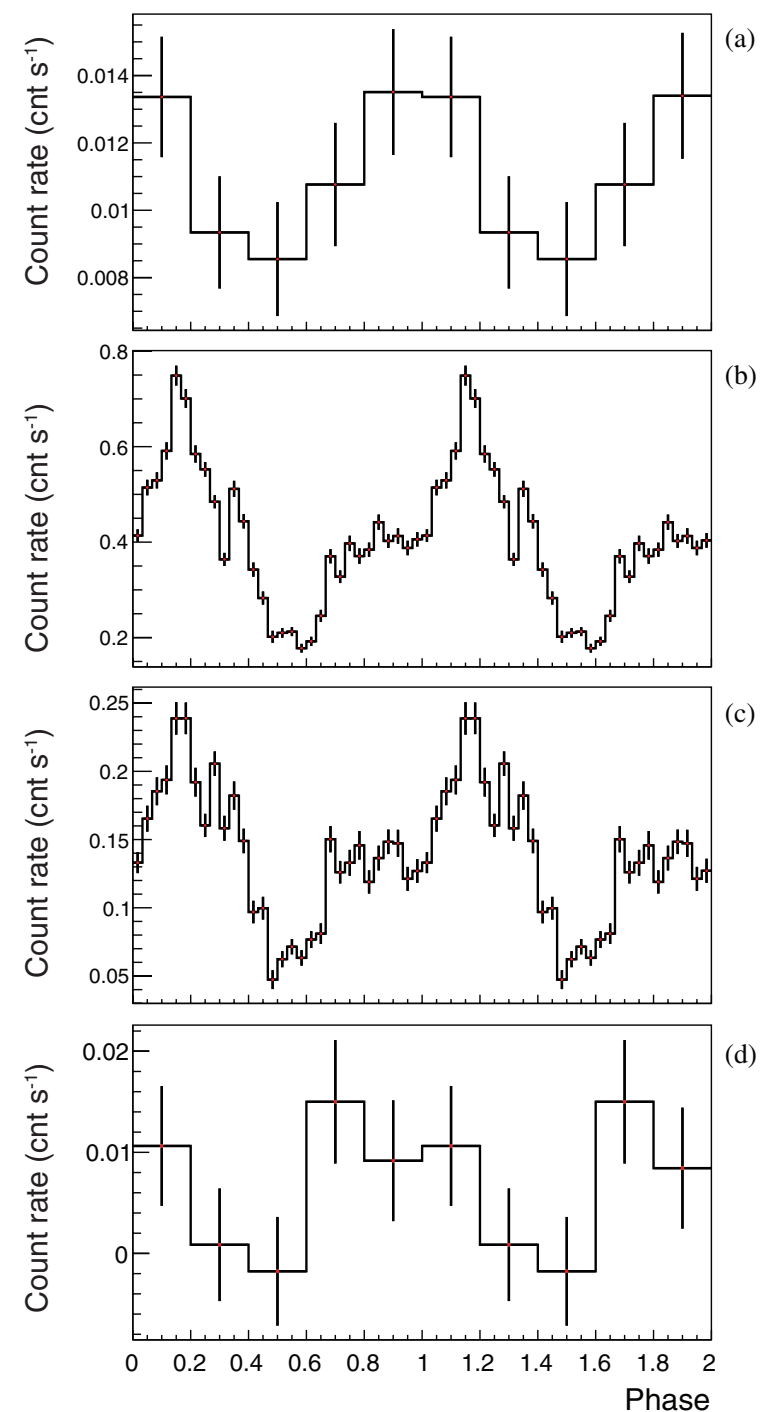

(c)
Suzaku 2012
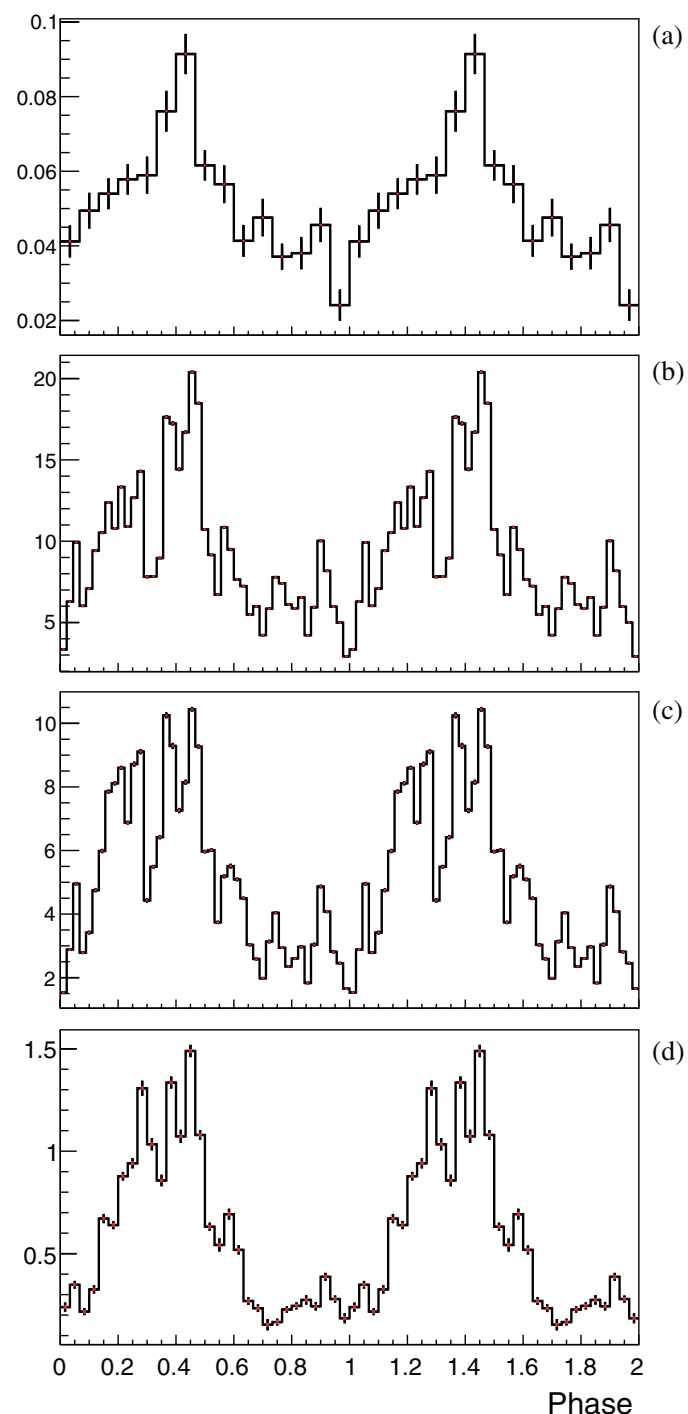

Figure 6. Pulse profiles of $4 \mathrm{U} 1954+319$ folded with $5.70 \mathrm{hr}$ and $5.76 \mathrm{hr}$ in 2011 (left) and 2012 (right), respectively. Panels (a), (b), (c), and (d) represent the

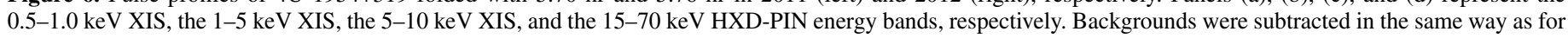
Figure 3.

(A color version of this figure is available in the online journal.)

Table 2

Comparison of the 2012 and 2011 Time-averaged Spectra of 4U 1954+319

\begin{tabular}{|c|c|c|c|c|c|c|c|c|}
\hline Model & $\begin{array}{c}N_{\mathrm{H}} \\
\left(10^{22} \mathrm{~cm}^{-2}\right)\end{array}$ & $\Gamma$ & $\begin{array}{c}E_{\text {cut }} \\
(\mathrm{keV})\end{array}$ & $\begin{array}{c}E_{\text {fold }} \\
(\mathrm{keV})\end{array}$ & $\begin{array}{c}k T_{e} \\
(\mathrm{keV})\end{array}$ & $\begin{array}{c}k T \\
(\mathrm{keV})\end{array}$ & $\tau$ & $\chi^{2} /$ dof (Prob.) \\
\hline \multicolumn{9}{|c|}{2012 AO7 ToO observation } \\
\hline bbody+cutoffpl & $5.4 \pm 0.1$ & $1.21 \pm 0.01$ & $\cdots$ & $19.9 \pm 0.5$ & $\cdots$ & $0.13 \pm 0.01$ & $\cdots$ & $971.5 / 696\left(2.0 \times 10^{-11}\right)$ \\
\hline bbody+pow*highecut & $4.9 \pm 0.1$ & $1.24 \pm 0.01$ & $4.23_{-0.19}^{+0.17}$ & $21.0_{-0.8}^{+0.9}$ & $\cdots$ & $0.13 \pm 0.01$ & $\cdots$ & $890.1 / 696\left(6.9 \times 10^{-7}\right)$ \\
\hline bbody+NPEX & $4.9 \pm 0.1$ & $0.77 \pm 0.01$ & $6.6 \pm 0.1$ & $\ldots$ & $\ldots$ & $0.14 \pm 0.01$ & $\ldots$ & $868.8 / 695\left(7.2 \times 10^{-6}\right)$ \\
\hline compTT & $1.7 \pm 0.2$ & $\cdots$ & $\cdots$ & $\cdots$ & $7.5_{-0.1}^{+0.2}$ & $1.00 \pm 0.01$ & $8.9_{-0.14}^{+0.13}$ & $780.8 / 697(0.015)$ \\
\hline \multicolumn{9}{|c|}{2011 AO6 observation } \\
\hline bbodyrad & $1.5 \pm 0.1$ & $\cdots$ & $\cdots$ & $\cdots$ & $\ldots$ & $1.28 \pm 0.01$ & $\ldots$ & $162.7 / 124(0.01)$ \\
\hline diskbb & $3.0 \pm 0.1$ & $\ldots$ & $\cdots$ & $\ldots$ & $\cdots$ & $2.19 \pm 0.03$ & $\cdots$ & $162.3 / 124(0.12)$ \\
\hline cutoffpl & $2.1_{-0.1}^{+0.2}$ & $-0.73 \pm 0.17$ & $\ldots$ & $1.9 \pm 0.1$ & $\cdots$ & $\ldots$ & $\cdots$ & $133.9 / 123(0.23)$ \\
\hline pow*highecut & $2.0 \pm 0.2$ & $0.28_{-0.20}^{+0.19}$ & $3.0 \pm 0.1$ & $3.0 \pm 0.3$ & $\ldots$ & $\ldots$ & $\ldots$ & $132.4 / 122(0.24)$ \\
\hline compTT & $1.6 \pm 0.1$ & $\cdots$ & $\ldots$ & $\ldots$ & $<5.1$ & $0.85_{-0.04}^{+0.10}$ & $15.3_{-8.8}^{+0.7}$ & $129.0 / 122(0.31)$ \\
\hline
\end{tabular}

Notes. All the spectral uncertainties are given at $1 \sigma$ confidence level for single parameters. The photo-absorption and Gaussian components are included in all the spectral models. The parameter $E_{\text {cut }}$ in the xspec cutoffpl model is actually a folding energy, and shown in the $E_{\text {fold }}$ column to compare the highecut model. 


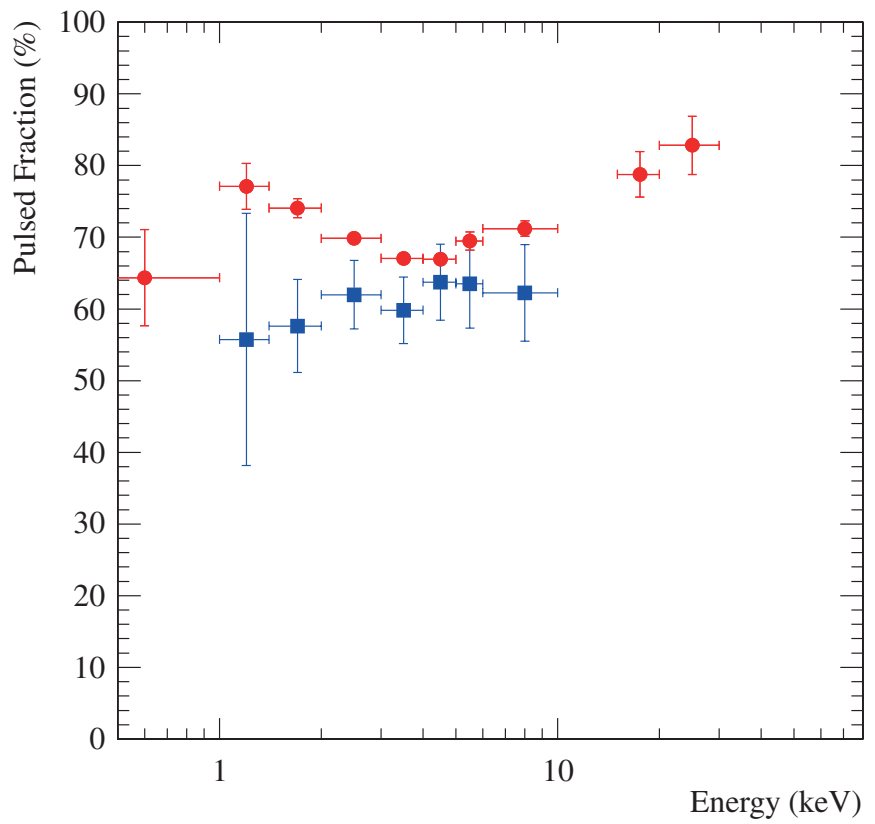

Figure 7. Pulsed fraction of 4U 1954+319 in 2011 (blue) and 2012 (red).

(A color version of this figure is available in the online journal.)

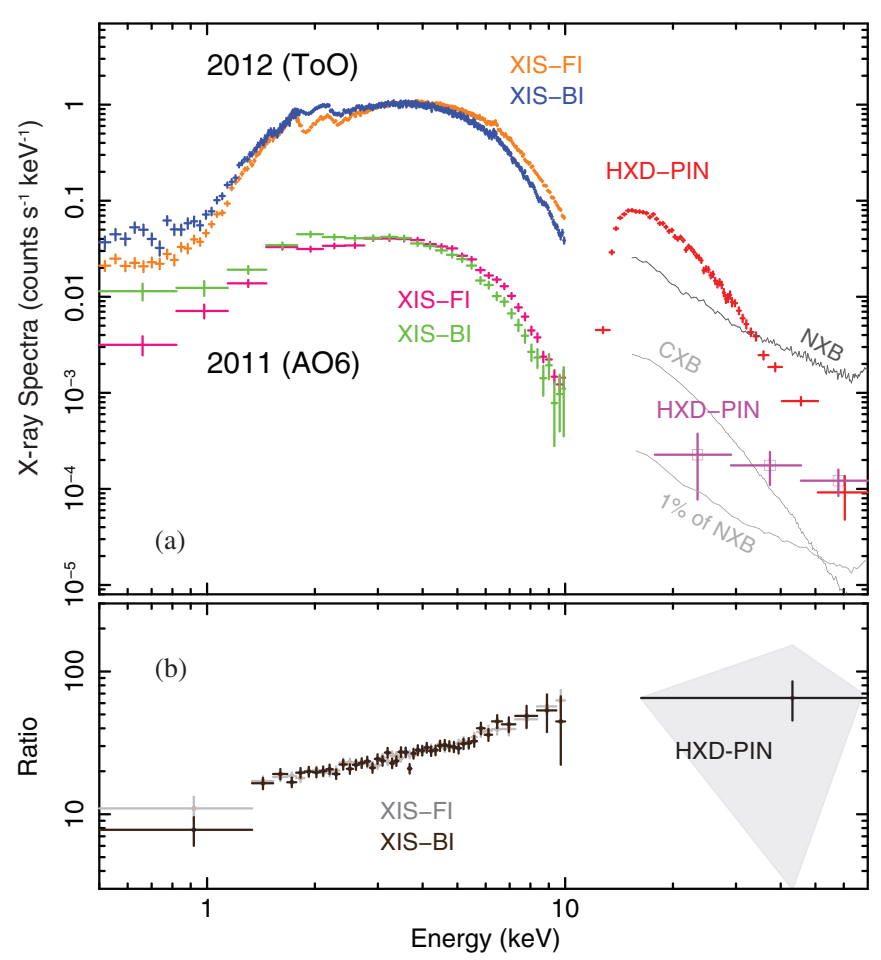

Figure 8. (a) Time-averaged, background-subtracted raw XIS-FI, XIS-BI, and HXD-PIN spectra of 4U 1954+319 during the two Suzaku pointings in count rate space. The modeled NXB, CXB, and $1 \%$ of NXB (a typical uncertainty of the NXB modeling) are also indicated. (b) Spectral ratio of the $2012 \mathrm{ToO}$ data to those from the 2011 observation. The gray diamond of the HXD-PIN point represents an error region including $0.3 \%$ systematic uncertainties.

(A color version of this figure is available in the online journal.)

of $\left.2.1 \times 10^{-4}\right)$. Then, when we try various abundance tables (e.g., angr; Grevesse \& Anders 1989; aspl, Asplund et al. 2009) with the phabs absorption model (Balucinska-Church \& McCammon 1992), the same comptt continuum changes $N_{\mathrm{H}}$ up to by $\sim 50 \%$. Under these uncertainties, we assume

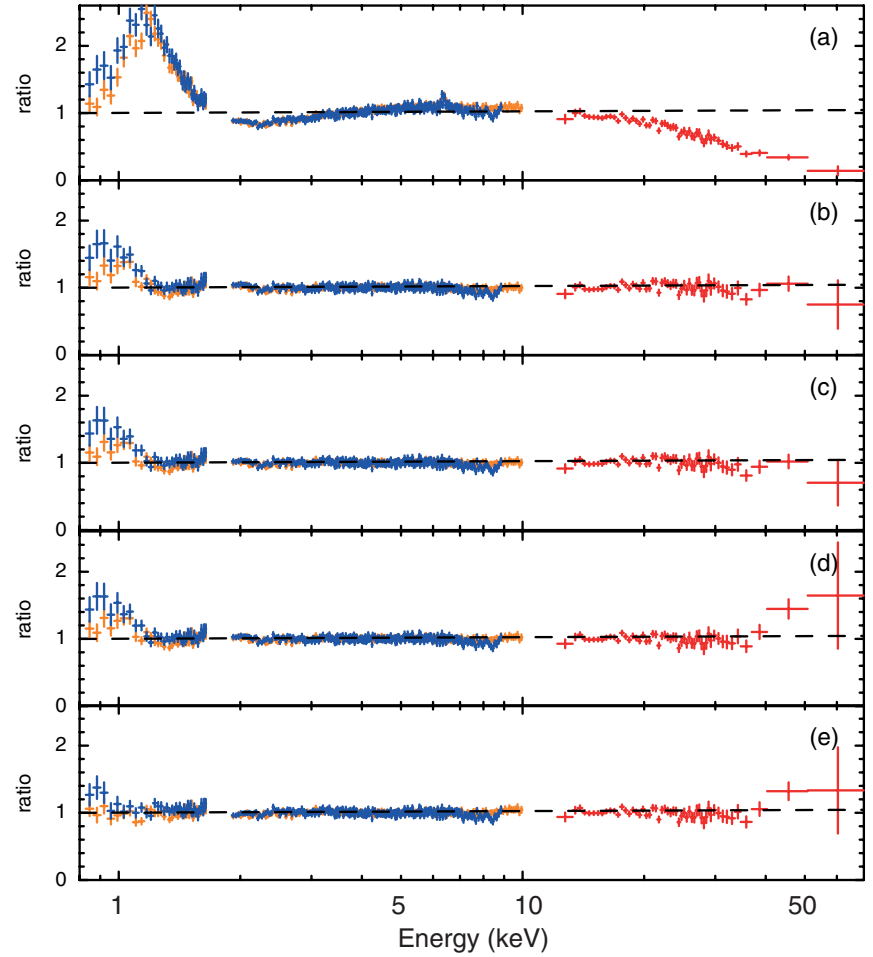

Figure 9. Ratios of the best-fit spectral model to the 2012 Suzaku data, using (a) a single power law, (b) a cutoff power law, (c) a high energy cutoff, (d) the NPEX, and (e) the comptt. The $6.4 \mathrm{keV}$ Gaussian emission is included in panels (b)-(e), while a soft blackbody component $(k T \sim 0.13 \mathrm{keV})$ is added in panels (b)-(d).

(A color version of this figure is available in the online journal.)

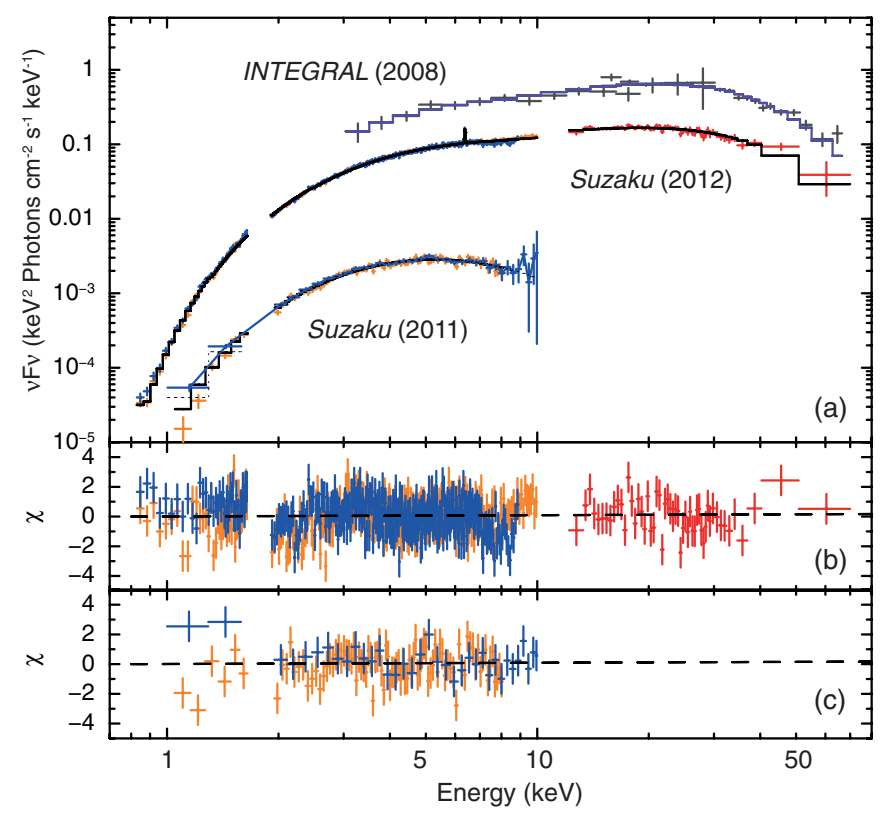

Figure 10. (a) X-ray spectra of $4 \mathrm{U} 1954+319$ in $v F_{v}$ form observed during the 2011 quiescent and 2012 flare-up, utilizing the comptt continuum (Model C). For comparison, INTEGRAL spectra, JEM-X (3-30 keV) and ISGRI (20-80 keV) for the flaring episode in 2008, are overlaid with a comptt model fit as used by Marcu et al. (2011), (b) residuals of the comptt model in 2012. (c) The same as panel (b) but for the 2011 data.

(A color version of this figure is available in the online journal.) 
$N_{\mathrm{H}}=1.7 \times 10^{22} \mathrm{~cm}^{-2}$, derived from the best-fit comptt model, as the default value in the discussion. ${ }^{14}$ Subtracting the $21 \mathrm{~cm}$ interstellar column density in the direction to $4 \mathrm{U} 1954+319$, $N_{\mathrm{H}}=0.89 \times 10^{22} \mathrm{~cm}^{-2}$ (Kalberla et al. 2005), the intrinsic column density in the binary system is $N_{\mathrm{H}} \sim 0.81 \times 10^{22} \mathrm{~cm}^{-2}$.

We also search the HXD-PIN data for a possible presence of a cyclotron resonance scattering feature (CRSF), which provides a direct probe of the $B$-field at the accretion column (Makishima et al. 1999; Schönherr et al. 2007). Although a shallow structure at $\sim 40 \mathrm{keV}$ is visible, the depth of the absorption (cyclabs) was constrained to $D<1.1(1 \sigma)$. Thus, no CRSF was found in the Suzaku data.

\subsection{X-Ray Spectrum in the 2011 Quiescent State}

In the first Suzaku observation performed in 2011 before the flare, the source was fainter by more than an order of magnitude, with the absorbed 2-10 keV X-ray flux $5.36 \pm$ $0.03 \times 10^{-12} \mathrm{erg} \mathrm{s}^{-1} \mathrm{~cm}^{-2}$ (comptt model). Since the HXD-PIN detection is marginal, we only took the XIS-FI and BI spectra into account for this data set. Table 2 summarizes the spectral models used to fit the data. Simple power-law and blackbody models failed with $\chi_{v}^{2}>1.3$, while the diskbb, cutoffpl, pow*highecut, and compTT models were all acceptable. Using the same comptt model, the unabsorbed $1-10 \mathrm{keV}$ flux was $6.38 \pm 0.09 \times 10^{-12} \mathrm{erg} \mathrm{s}^{-1} \mathrm{~cm}^{-2}$. The $1 \sigma$ upper-limit intensity of a narrow $(\sigma=10 \mathrm{eV}) \mathrm{Fe}-\mathrm{K} \alpha$ line at $6.4 \mathrm{keV}$ was $I_{\mathrm{Fe}}<9.8 \times 10^{-7}$ photon $\mathrm{cm}^{-2} \mathrm{~s}^{-1}$.

\subsection{Luminosity and Mass Accretion Rate}

As seen above, between the quiescent phase in 2011 and the outburst phase of 2012 the time-averaged X-ray flux of $4 \mathrm{U} 1954+319$ changed by a factor of about 100. Assuming a distance of $1.7 \mathrm{kpc}$, the fluxes observed in 2011 and 2012 correspond to $1-70 \mathrm{keV}$ luminosities of $0.022 L_{35}$ and $2.1 L_{35}$ with $L_{35}=10^{35} \mathrm{erg} \mathrm{s}^{-1}(d / 1.7 \mathrm{kpc})^{2}$. Correcting for the X-ray absorption increases these luminosities to $0.025 L_{35}$ and $2.2 L_{35}$, respectively. These values fall in the luminosity range typically seen in SyXBs, $L_{\mathrm{x}} \sim 10^{33}-10^{35} \mathrm{erg} \mathrm{s}^{-1}$ (Lü et al. 2012).

We can estimate the accretion rate onto the NS as

$$
\dot{M}_{\mathrm{NS}}=\frac{R_{\mathrm{NS}}}{\eta G M_{\mathrm{NS}}} L_{\mathrm{X}}=\left\{\begin{array}{ll}
4.7 \times 10^{13} \mathrm{~g} \mathrm{~s}^{-1} & \text { in } 2011 \\
3.8 \times 10^{15} \mathrm{~g} \mathrm{~s}^{-1} & \text { in } 2012
\end{array},\right.
$$

where $\eta=0.3$ is the efficiency of accretion, $R_{\mathrm{NS}}=10 \mathrm{~km}$ is the NS radius, and $M_{\mathrm{NS}}=1.4 M_{\odot}$ is the NS mass.

Since 4U 1954+319 is a highly variable source (Section 3.2), we further investigated, in Figure 11, distributions of count rates in the XIS and HXD-PIN light curves, referring to Vela X-1 studies by Fürst et al. (2010). The count rate distributions closely follow a log-normal distribution in both the quiescent phase (2011, Figure 11(a)) and the outburst (2012, Figures 11(a) and (b)). Assuming the 2012 time-averaged spectral shape, we further converted these to luminosity at the top of Figure 11(a). As previously shown in Figure 8, the spectrum becomes harder as the X-ray luminosity increases from the quiescent phase (2011) to the outburst (2012). Such a hardening trend is also detected within the 2012 observation when we divide the data into three intensity-sorted spectra (Figure 12).

\footnotetext{
14 If the $\mathrm{Fe}-\mathrm{K} \alpha$ line is due to fluorescence from an optically thin gas which spherically surrounds the source, its equivalent width is expected to be linearly correlated with the column density, $N_{\mathrm{H}}$ as $\mathrm{EW}=100\left(N_{\mathrm{H}} / 10^{23} \mathrm{~cm}^{-2}\right.$ ) (Inoue 1985). The line could therefore be produced in a gas with a column density of $N_{\mathrm{H}}=2.9 \times 10^{22} \mathrm{~cm}^{-2}$.
}

\section{DISCUSSION}

\subsection{Summary of the Observations}

Combining the Swift/BAT and RXTE/ASM long-term monitoring with the Suzaku observations, we derived the following observational results which need to be explained.

1. The slow $5.4 \mathrm{hr}$ NS spin period of $4 \mathrm{U} 1954+319$ exhibits a $\sim 7 \%$ fluctuation over $\sim 8 \mathrm{yr}$ with at least four reversals in the sign of the period derivative.

2. In the 2011 October and 2012 November Suzaku observations, before and after the 2012 November X-ray outburst, the $1-70 \mathrm{keV} X$-ray luminosities were $2.2 \times 10^{33} \mathrm{erg} \mathrm{s}^{-1}$ and $2.1 \times 10^{35} \mathrm{erg} \mathrm{s}^{-1}$, respectively. In the former and the latter observations, the source was in a spin-down and a spin-up phase, respectively.

3. The $0.5-70 \mathrm{keV} X$-ray pulse profile shows a main peak and a sub peak. The PF mostly increases with energy from $\sim 60 \%$ at $3 \mathrm{keV}$ to $80 \%$ at $30 \mathrm{keV}$.

4. During the 2012 outburst, the X-ray flux was highly variable on 10-1000 s timescales, with many irregular short flares each with a typical duration of $\lesssim 100$ s. The Fourier power density spectrum is dominated by red noise, i.e., became redder from -0.77 in 2011 to -1.66 in 2012 . The X-ray count rates follow a log-normal distribution in both observations.

5. The $0.8-70 \mathrm{keV}$ phase-averaged spectra in the 2012 outburst can be represented by the Comptonization spectral models; compTT, BB+NPEX, or BB+highecut. A fluorescent $\mathrm{Fe}-\mathrm{K} \alpha$ line at $6.37 \mathrm{keV}$ is detected with an $\mathrm{EW}$ of $\mathrm{EW}=28.6 \pm 0.3 \mathrm{eV}$, and a line flux of $I_{\mathrm{Fe}}=$ $7.5_{-0.7}^{+0.8} \times 10^{-5}$ photons $\mathrm{cm}^{-2} \mathrm{~s}^{-1}$. The line is narrow, with a $1 \sigma$ upper limit for the Gaussian width of $50 \mathrm{eV}$. The quiescent 2011 spectrum is also well described by comptt with $k T \sim 0.85 \mathrm{keV}$, or also by diskbb, cutoffpl, and highecut models.

6. The 2012 XIS spectrum is harder than that measured in 2011. The intensity-sorted X-ray spectra in 2012 slightly hardens with source brightness.

7. The $1-70 \mathrm{keV}$ band does not contain any statistically significant cyclotron lines.

\subsection{Are SyXBs a Subclass of LMXB?}

X-ray binaries hosting NSs are conventionally classified based on stellar types of the optical counterparts. Commonly, HMXBs host a strongly magnetized NS and a high-mass companion (i.e., Be stars or OB supergiants), while LMXBs are thought to host a weakly magnetized NS and a low-mass star (e.g., K-type stars). However, this old optical identification ignores accretor properties (e.g., NS $B$-field) which mainly determine the X-ray radiation. In fact, the conventional stereotype assuming weakly magnetized NSs in LMXBs is now challenged by recent observational evidence of strongly magnetized NSs in LMXBs, e.g., 4U 1626-67 (Camero-Arranz et al. 2012) and 4U 1822-37 (Sasano et al. 2013).

The required overhaul of this outworn notion is more clearly shown in SyXBs. In contrast to the conventional classification which makes SyXBs the same category as LMXBs, the apparent high PF (60\%-80\%) of SyXBs (Figure 7, Section 4.1, point 3) suggests a similarity to strongly magnetized and highly pulsed NSs in HMXBs (PF 20\%-100\%; Lutovinov \& Tsygankov 2008) rather than to non-pulsed NSs in LMXBs or to weakly pulsed millisecond X-ray pulsars (typical PF at a few percent; 

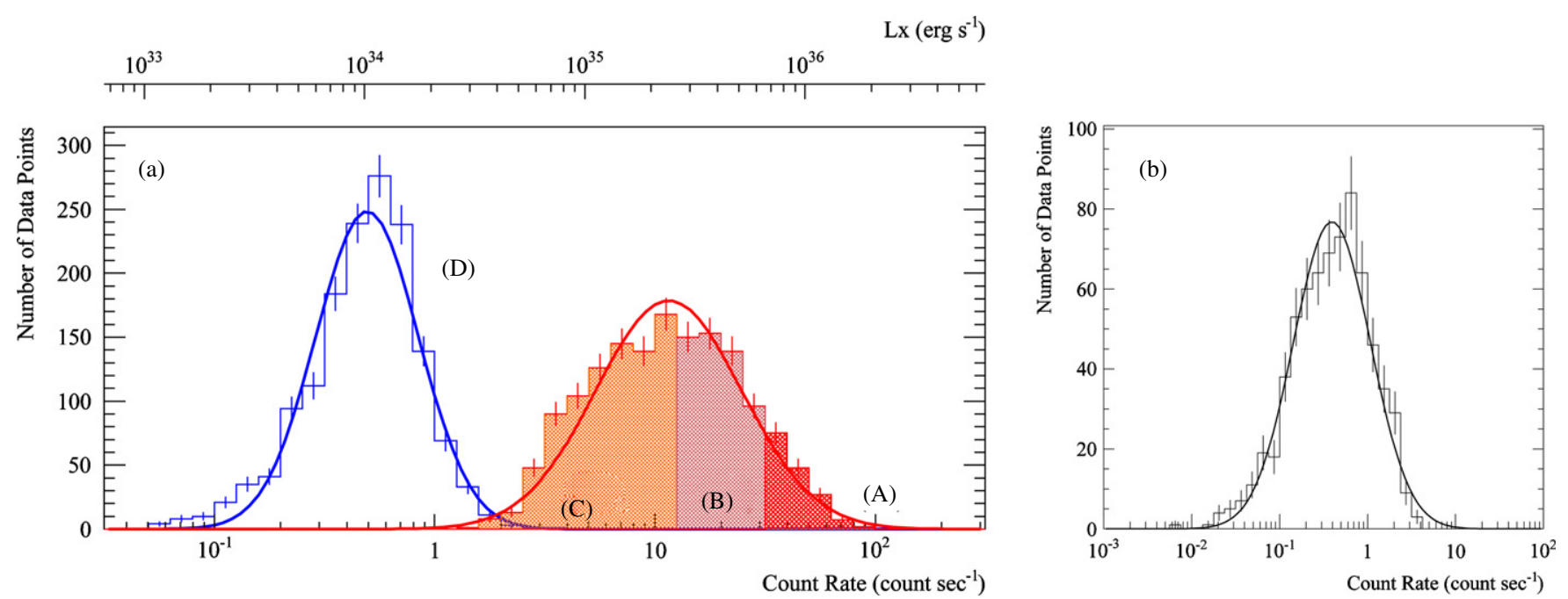

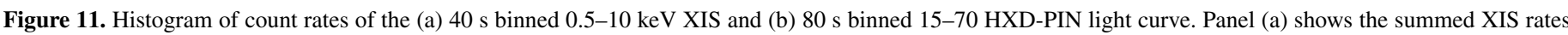

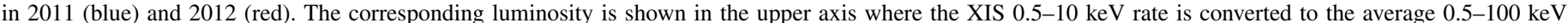

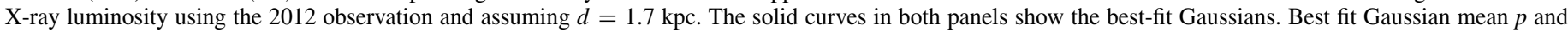

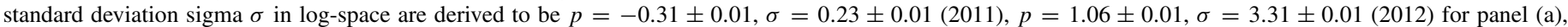

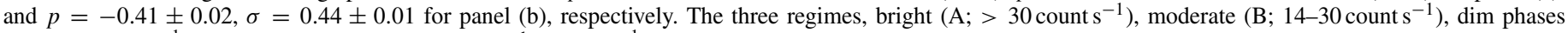
$\left(\mathrm{C} ;<14\right.$ count s$\left.^{-1}\right)$ and the 2011 quiescent state $\left(\mathrm{D} ; \lesssim 2\right.$ count s$\left.^{-1}\right)$ are shaded.

(A color version of this figure is available in the online journal.)

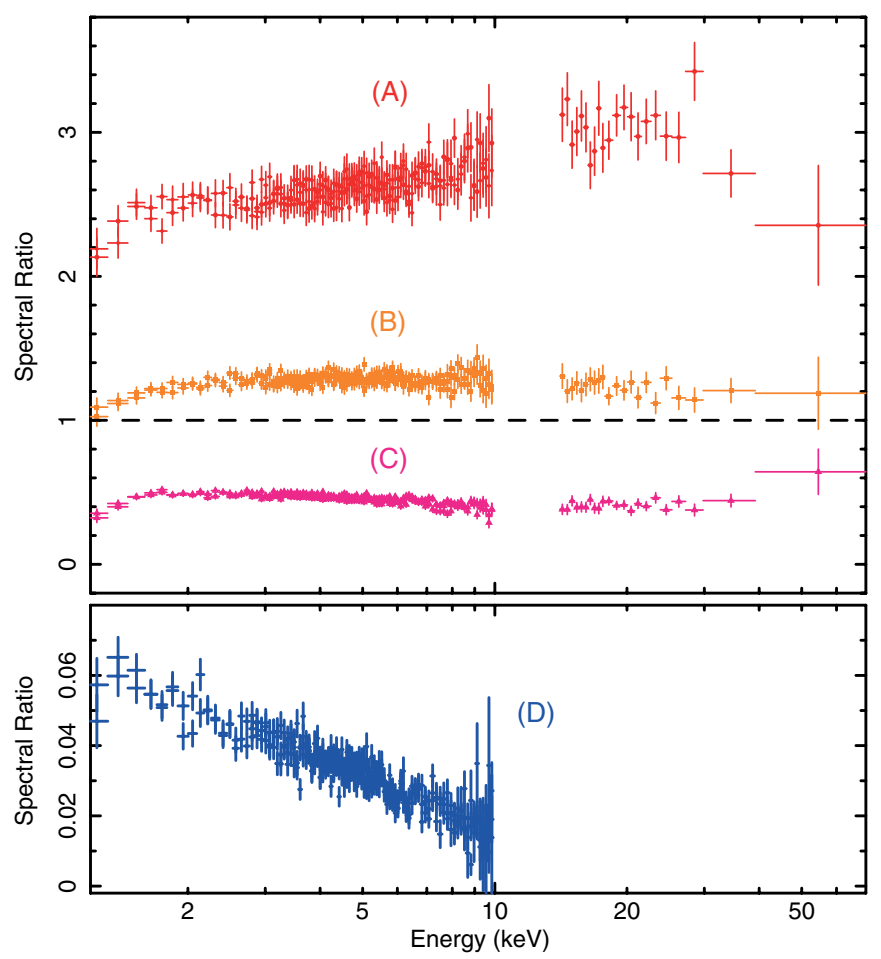

Figure 12. Spectral ratios of $4 \mathrm{U} 1954+319$ to the average one in 2012. Four different X-ray luminosity states, (A), (B), (C), and (D) which were defined in Figure 11 .

(A color version of this figure is available in the online journal.)

Patruno \& Watts 2012). In addition, if we look at the location of SyXBs in the "Corbet diagram" (Corbet 1986) shown in Figure 13, SyXBs again resemble HMXBs due to their wideorbit and slow NS rotation. Finally, SyXB X-ray spectra and that of $4 \mathrm{U} 1954+319$, in particular, and the other behavior summarized in Section 4.1 also suggest a closer similarity of $4 \mathrm{U} 1954+319$ to X-ray pulsars in HMXBs with a $10^{12}-10^{13} \mathrm{G}$ $B$-field.
Even though many of these points argue toward a closer similarity of SyXB and $\mathrm{HMXB}$, such an association would be a significant challenge to our current understanding of $\mathrm{X}$-ray binary evolution. Stellar evolution theory shows that the evolutionary timescale for the companion of the NS to become an M4-5 III counterpart is much longer than the typical lifetime of binary systems with OB-type donors, and thus, the $4 \mathrm{U}$ $1954+319$ system would be old. If one dogmatically expected NS $B$-field decay, the high $B$-field indicated by the strong X-ray pulsation easily causes a contradiction to the assumption of an old binary system. Furthermore, it is also difficult to keep such a binary system after a supernovae explosion, since the common envelope hypothesis, which is usually assumed to make close binary systems hosting NSs in LMXBs, cannot be reconciled with the suggested large orbit of SyXBs (Figure 13). Te conventional evolutional theory leaves us with a real conundrum.

In addition to needing to study the population synthesis of such binaries (see, e.g., Lü et al. 2012; Chakrabarty \& Roche 1997), the observed nature of SyXB requires us to investigate more exotic scenarios, e.g., a magnetar-descendent was captured by an evolved M-type giant in a close encounter via a magnetic braking interaction between the NS strong $B$-field and a large atmosphere of the M-type giant. An alternative possibility is an accretion-induced collapse of a white dwarf (Nomoto \& Kondo 1991) which potentially produces not only weak-field pulsars but also a magnetar (Thompson \& Duncan 1995).

In the following, we take the features of 4U 1954+319 listed in Section 4.1 as observational clues to the mystery of SyXB, comparing the conventional accretion model and the revisited quasi-spherical accretion model, regardless of the conventional HMXB and LMXB classification and population synthesis.

\subsection{The X-Ray Spectrum of $4 U 1954+319$}

The first clues on the nature of 4U 1954+319 come from broadband spectroscopy. We assume that the observed X-ray spectrum is uniquely characterized mainly by $\dot{M}$, $B$-field, and inclination of the compact object regardless of the 


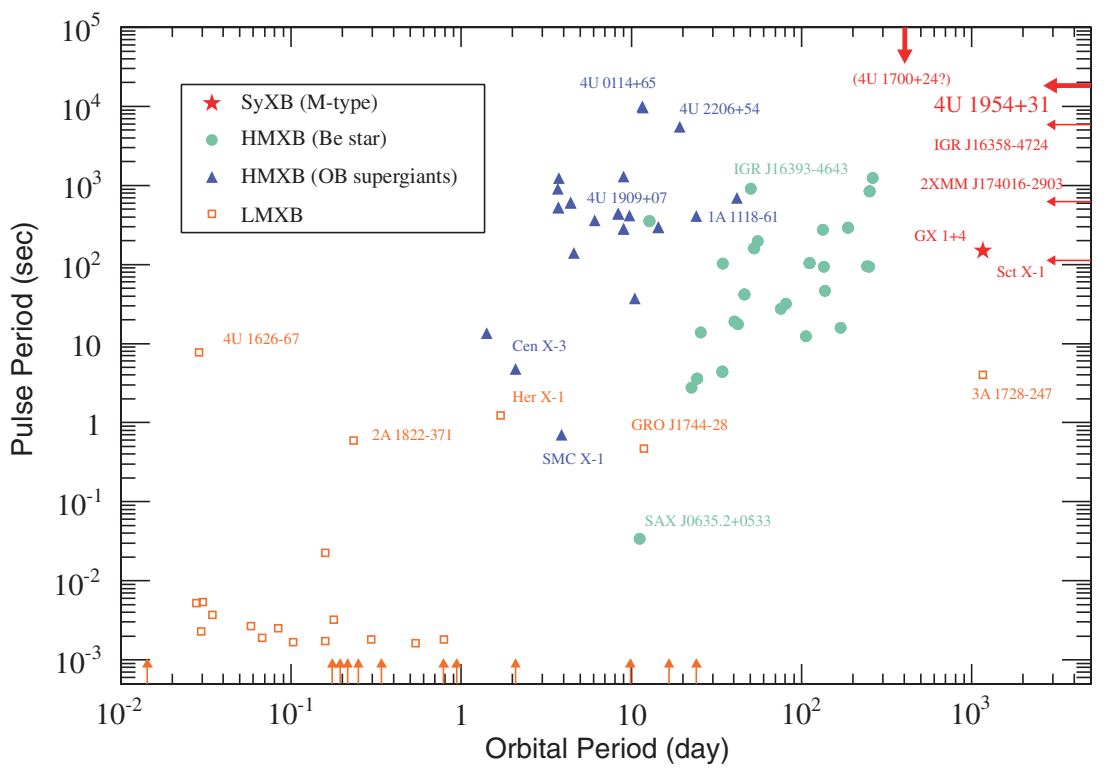

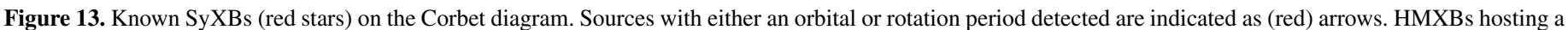

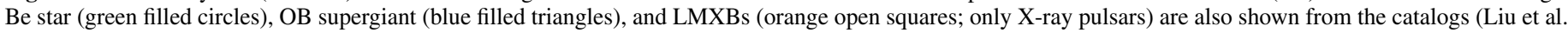

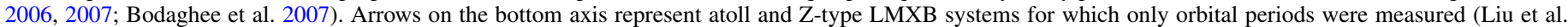
2007).

(A color version of this figure is available in the online journal.)

type of the mass-donor. As shown in Section 2.3 and previously suggested by Masetti et al. (2007b), spectral modeling implies that Comptonization is the dominant process forming the $\mathrm{X}$-ray continuum, similar to the accretion column of the canonical X-ray pulsars in HMXBs. ${ }^{15}$ Thus, in Figure 14, we now compare the X-ray spectrum of 4U 1954+319 with different $\mathrm{X}$-ray spectral shapes of other related X-ray pulsars.

Figure 14(a) shows a comparison of the X-ray spectrum of 4U 1954+319 with all archival Suzaku SyXB observations: GX 1+4, 4U 1700+24 (Nagae et al. 2008), and IGR J16194-2810 (Kitamura et al. 2014). The rotational, orbital, and optical information of these X-ray sources is listed in Table 3 with the Suzaku observation records and references. To extract these spectra, we use the same procedure as for $4 \mathrm{U} 1954+319$ (Section 2.2). The $v F_{v}$ spectra were obtained by fitting the data with a cutoffpl model with interstellar absorption (phabs) and, if needed, an iron line. GX 1+4 exhibits harder spectra peaking at $\sim 20-40 \mathrm{keV}$ than $4 \mathrm{U} 1954+319$. The spectral shape of IGR J16194-2810, for which orbital and pulse periods have not yet been detected, is similar to that of $4 \mathrm{U} 1954+319$ in quiescence, and thus, their accretion environment is expected to be similar.

In many accreting NSs with $B$-fields in the $10^{12} \mathrm{G}$ regime, cyclotron lines at energies $E_{\text {cyc }}=11.6\left(B_{\text {cyc }} / 10^{12} \mathrm{G}\right)(1+z)^{-1} \mathrm{keV}$ have been seen, where $z \sim 0.2$ is the gravitational red shift of the NS (Caballero \& Wilms 2012; Schönherr et al. 2007). In Figure 14(b), we compare 4U 1954+319 with well-studied CRSF sources. The $1.24 \mathrm{~s}$ X-ray pulsar Her X-1 is a prototypical CRSF source with $E_{\text {cyc }} \sim 36 \mathrm{keV}\left(B \sim 3.1 \times 10^{12} \mathrm{G}\right)$ with an unusual optical companion (A9-B companion with a mass of $\left.M_{\mathrm{c}} \sim 2.3 M_{\odot}\right)$. The HMXBs 1A 1118-616 and A $0535+262$ exhibit the strongest $B$-fields among known CRSF

\footnotetext{
15 Note that previous observations also included a soft excess which could be described with plasma emission with $k T \sim 50 \mathrm{eV}$ (Masetti et al. 2007b), which is not seen here since the Suzaku low energy data below $\sim 1 \mathrm{keV}$ have uncertainties due to the contamination on the optical blocking filter.
}

sources, reaching $B \sim 4.7 \times 10^{12} \mathrm{G}$ and $\sim 4.1 \times 10^{12} \mathrm{G}$, respectively. IGR J16393-4643 was originally classified as a SyXB but was finally identified as a HMXB (Bodaghee et al. 2012; Pearlman et al. 2011). As shown in Figure 14(b), the spectral shape of $4 \mathrm{U} 1954+319$ is similar to $1 \mathrm{~A} 1118-616$ and A $0535+262$ rather than Her X-1. Although a widely accepted physical model has not yet been established to explain the $\mathrm{X}$-ray continuum, it has been suggested that due to cyclotron cooling the high energy cutoff appears around the CRSF energy. This means that the X-ray continuum is expected to extend to higher energies as the $B$-field becomes stronger (Makishima et al. 1999).

The non-detection of a CRSF in 4U 1954+319 with the XIS and HXD means that either the $B$-field is not in the range $B=9.0 \times 10^{10}-6.0 \times 10^{12} \mathrm{G}$, or that the cyclotron line is too faint to be detected in the data. The latter is not too unlikely, with a number of sources with similar behavior being observed. A possible explanation for this is that CRSFs can be smeared out and/or filled by photon spawning, i.e., the emission of photons close to the resonance energy during their de-excitation from higher Landau levels (Schönherr et al. 2007; Fürst et al. 2011a). If, on the other hand, $B \gtrsim 10^{13} \mathrm{G}$, according to Makishima et al. (1999) we would expect a harder X-ray continuum than that of HMXBs with a cyclotron line.

In Figure 14(c), we compare the spectrum of 4U 1954+319 with those of some HMXBs, which have not shown a clear CRSF: HMXB 4U 1909+07, 4U 2206+54, and 4U 0114+65. The phase-averaged spectral shapes of these sources are quite similar to that of $4 U 1954+319$. From a spectral view point, they can be regarded as triplets. $4 \mathrm{U} 1909+07$ is a HMXB with an OB companion hosting a $604 \mathrm{~s}$ X-ray pulsar in a 4.4 day orbit. Its pulse period is reported to show a random walk-like behavior (Fürst et al. 2011a), which also resembles the period history of 4 U 1954+319 and possibly suggests wind type accretion rather than the stable accretion disk. The other two X-ray pulsars, $4 \mathrm{U}$ $2206+54$ and $4 U$ 0114+65, exhibit very long rotational periods of $\sim 5500 \mathrm{~s}$ and $\sim 9700 \mathrm{~s}$, respectively (Finley et al. 1992; 

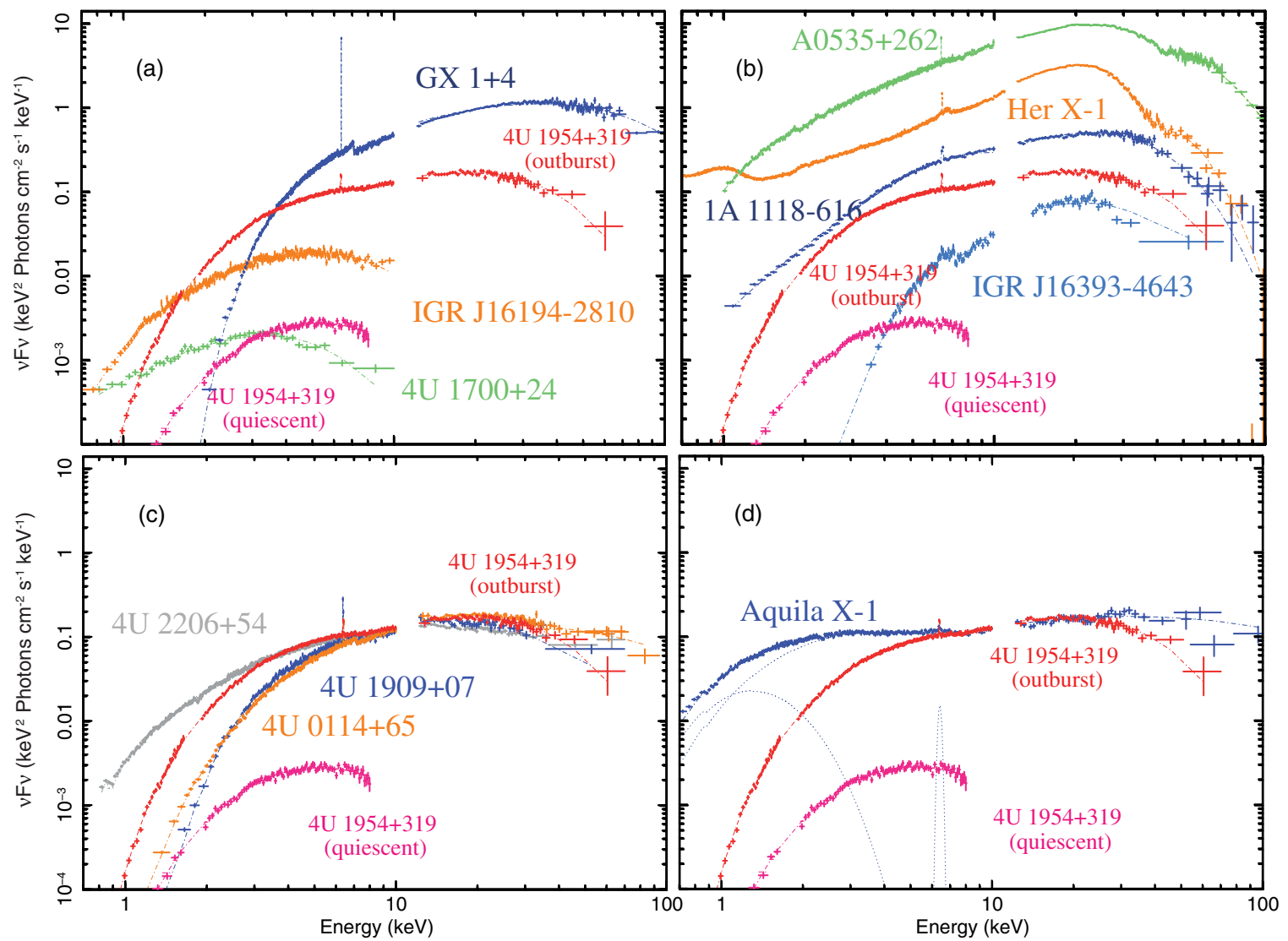

Figure 14. Spectral comparison of $4 \mathrm{U} 1954+319$ with different categories of NS binaries: (a) other SyXBs, (b) CRSF sources, (c) long period pulsars, and (d) the LMXB Aquila X-1 (Sakurai et al. 2012) in the low/hard state.

(A color version of this figure is available in the online journal.)

Table 3

Archival Suzaku X-Ray Pulsars Comapred with 4U 1954+319

\begin{tabular}{lcccccc}
\hline \hline X-ray source & $\begin{array}{c}P_{\mathrm{s}} \\
(\mathrm{sec})\end{array}$ & $\begin{array}{c}P_{\text {orb }} \\
\text { (day) }\end{array}$ & $\begin{array}{c}\text { Optical } \\
\text { Type }\end{array}$ & ObsID & $\begin{array}{c}\text { Exp } \\
(\mathrm{ks})\end{array}$ & Note \\
\hline 4U 1954+31 & 18300 & $? ?$ & M4 III & (Table 1) & $60.2 / 60.8$ & this work \\
GX 1+4 & 150 & 1161 & M5 III & 405077010 & 97.2 & cutoffpl [1] \\
4U 1700+24 & $? ?$ & $(404 ?)[2]$ & M2 III & 402023010 & 50.3 & cutoffpl [3] \\
IGR J16194-2810 & $? ?$ & $? ?$ & M2 III & 403024010 & 45.6 & cutoffpl [4] \\
\hline Her X-1 & 1.24 & 1.70 & A9-B & 100035010 & 36.9 & cutoffpl+cutoffpl [5] \\
1A 1118-616 & 408 & 24 & O9.5 & 403050010 & 22.0 & cutoffpl \\
A 0535+262 & 103 & 111 & O9.7 & 404055010 & 31.8 & highecut \\
IGR J16393-4643 & 912 & 50.2 & B? [6] [7] & 404056010 & 50.6 & cutoffpl [8] \\
\hline 4U 0114+65 & 9720 & 11.63 & B1Ia & 406017010 & 106.6 & cutoffpl+cutoffpl \\
4U 2206+54 & 5555 & 19.25 & O9.5V & 402069010 & 51.6 & cutoffpl \\
4U 1909+07 & 604 & 4.4 & OB & 405073010 & 29.2 & cutoffpl \\
\hline Aql X-1 & $\cdots$ & 19.0 & K1 & 402053030 & 19.7 & bbody+compPS [9]
\end{tabular}

Notes. The photo-absorption is included in all the spectral models. Applied spectral models and references are noted. [1] Chakrabarty \& Roche 1997; [2] 404 day period in 4U 1700+24 was not confirmed in Corbet et al. 2008; [3] Masetti et al. 2006b; Nagae et al. 2008; [4] Masetti et al. 2007a; Kitamura et al. 2014; [5] Enoto et al. 2008; [6] Bodaghee et al. 2012; [7] Pearlman et al. 2011; [8] Nespoli et al. 2010; [9] Sakurai et al. 2012.

Reig et al. 2009; Masetti et al. 2006a; Farrell et al. 2008). Thus, we expect that such a long rotational period is strongly related to the spectral shape in Figure 14(c) of these sources.

Let us finally compare, in Figure 14(d), the spectrum with the low-luminosity $\left(L_{\mathrm{X}} \lesssim 10^{36} \mathrm{erg} \mathrm{s}^{-1}\right)$ low/hard state of LMXBs as motivated by Kitamura et al. (2014) who tried to search for similarities between SyXBs and low-field LMXB-NSs (e.g., Aql X-1) rather than high-field NSs, based on the optical classification. Although the $B$-field of such LMXBs starts to dominate the accretion flow and an optically thin spherical flow would realize a similar geometry as the SyXB, as we already discussed, SyXBs should be different from other LMXBs due to (1) slow pulsation with a large PF (Figure 6) suggesting a high $B$-field; (2) the large orbital period (Figure 13) suggesting windtype accretion; (3) the lack of clear evidence on the accretion disk; (4) no observations of LMXB-like strong soft blackbody 
spectra; and (5) the strong narrow $6.4 \mathrm{keV}$ iron fluorescence line, which is more typically found in HMXBs but rare $(\sim 10 \%)$ in standard LMXBs (Torrejón et al. 2010). In addition, LMXBs are usually characterized by highly ionized 6.6-6.9 keV lines.

In conclusion, the broadband 4U 1954+319 spectrum best resembles that of X-ray pulsars in HMXBs, especially slowly rotating pulsars (e.g., 4U 0114+65 or 4U1909+07) without an apparent accretion disk as well as another quiescent SyXB, IGR J16194-2810. Therefore, a strong field $\gtrsim 10^{12}$ or higher is favored rather than a low field as in non-pulsating or weakly pulsed NSs in LMXBs.

\subsection{The Long Spin Period of $4 U 1954+319$}

Let us now investigate the timing behavior of the source. As already discussed previously (e.g., Marcu et al. 2011; Corbet et al. 2008, and references therein), the $5.4 \mathrm{hr}$ period of $4 \mathrm{U}$ $1954+319$ can only be explained as being the rotation period of the NS. Both an orbital modulation or an M-type star pulsation, can be ruled out. The long-term pulse history implies at least four spin-torque transitions between a spin-up and a spin-down phase. The period evolution indicates that outside the outbursts the NS is gradually slowing down, while during the outbursts it enters a spin-up trend correlated with high accretion rates. This behavior suggests that it is reasonable to assume that the source is near an equilibrium period of accretion momentum transfer.

In the standard disk accretion model (Ghosh \& Lamb 1979), the equilibrium period is Equation (76) in Shakura et al. (2012)

$$
P_{\mathrm{eq}} \approx 7 \mu_{30}^{6 / 7} \dot{M}_{16}^{-3 / 7} \mathrm{~s}
$$

where $\dot{M}_{16}=\dot{M} /\left(10^{16} \mathrm{~g} \mathrm{~s}^{-1}\right)$ is the mass accretion rate, and $\mu_{30}=\mu /\left(10^{30} \mathrm{G} \mathrm{cm}^{3}\right)$ is the NSs magnetic dipole moment. Inserting the values for $4 \mathrm{U} 1954+319$ measured in 2012, i.e., $\dot{M}_{16}=0.38$ and $P_{\text {eq }} \sim 19400 \mathrm{~s}(\sim 5.4 \mathrm{hr})$ yields an extremely high, magnetar-like $B$-field reaching $B \sim 10^{16} \mathrm{G}$. This evaluation would hold even if considering the long-term history, since the average mass accretion rate is close to the value in the 2012 Suzaku observation. This is clearly shown in Figure 15, where the long-term distribution of mass accretion rates is illustrated estimated from Figure 2.

While a magnetar-like $B$-field has been suggested for the SyXB IGR J16358-4726 (Patel et al. 2007), the field derived above is an order of magnitude higher than the strongest magnetar field (SGR 1806-20, with $2.1 \times 10^{15}$ G; Kouveliotou et al. 1998; Enoto et al. 2010b). 4U 1954+319 also does not show any other magnetar-like properties, such as short bursts or giant flares (Enoto et al. 2009). In addition, it is unclear whether standard disk theory can be applied to SyXBs. For example, González-Galán et al. (2012) show that the spin and X-ray intensity behavior of the SyXB GX 1+4 cannot be explained either by standard disk accretion theory or even by more advanced ideas such as accretion from a retrograde disk. We therefore have to also consider alternative accretion models.

As the Corbet diagram (Figure 13) implies large orbital periods of SyXBs (e.g., $P_{\text {orb }}=1161 \mathrm{~d}$ for GX 1+4; Hinkle et al. 2006), it is reasonable to assume that $4 \mathrm{U} 1954+319$ does not accrete via Roche lobe overflow but is a wind-fed accretor. The large orbital period leads to a slow NS orbital velocity. ${ }^{16}$

\footnotetext{
16 Although the orbital radius of $4 \mathrm{U} 1954+319$ has not yet been detected, assuming a circular orbit with its orbital period at 500 days, we will derive the semimajor axis at $3 \times 10^{13} \mathrm{~cm}$ via the Kepler's law and masses of the NS and $\mathrm{M}$ giant. Then the orbital velocity becomes $v=\sqrt{G M / a} \sim 30 \mathrm{~km} \mathrm{~s}^{-1}$.
}

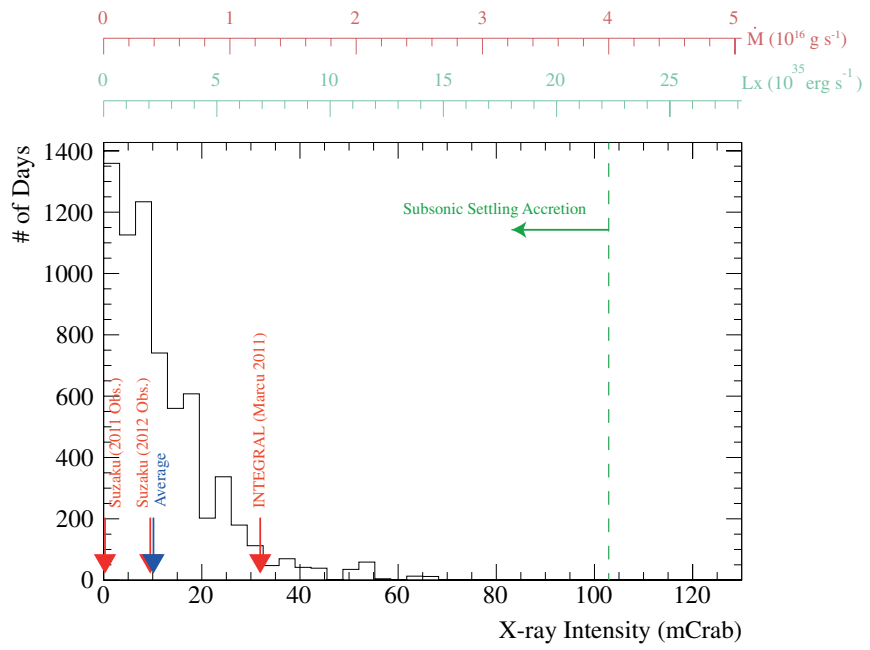

Figure 15. Histogram of the long-term 4U 1954+319 X-ray intensity in mCrab unit derived from Figure 2. Corresponding X-ray luminosity and mass accretion rate are shown in the upper axes, assuming the distance at $1.7 \mathrm{kpc}$ and $\eta=0.3$ See Table 1 for the conversion factor from mCrab to X-ray flux. The two Suzaku observations, one INTEGRAL observation (Marcu et al. 2011), and the average of the distributions are shown as arrows.

(A color version of this figure is available in the online journal.)

Combining this with a slow stellar wind compared to HMXBs and with a low mass accretion rate, small angular momentum differences are an intrinsic importance of the SyXBs which makes the mass accretion more quasi-spherical.

As recently shown by Shakura et al. (2013), the X-ray luminosity of $4 \mathrm{U} 1954+319$ actually puts the source in the regime of quasi-spherical, subsonic setting accretion in the magnetosphere. Figure 16 shows a sketch of this alternative wind accretion scenario for $4 \mathrm{U} 1954+319$, which is possible for $L_{\mathrm{X}} \leqslant L_{\mathrm{th}}=4 \times 10^{36} \mathrm{erg} \mathrm{s}^{-1}$ and $\dot{M}_{16} \leqslant 4$. Similar to the standard Bondi-Hoyle-Lyttleton accretion, in this scenario, a bow shock is formed in the wind at a characteristic distance $\left(R_{\mathrm{B}}\right)$ from the NS, and matter entering this region is accreted onto the compact object. Unlike the classical Bondi-Hoyle-Lyttleton accretion at higher luminosities, for the low $L_{X}$ of $4 U$ 1954+319, Compton cooling becomes ineffective, and the matter can settle in the magnetosphere with a subsonic velocity through a quasi-spherical hot shell (Section 1). If this happens, the angular momentum can be transferred via large-scale convective motions or turbulence in this quasi-static shell. This results in an equilibrium spin period of (Postnov et al. 2012)

$$
P_{\text {eq }} \simeq 13,000 \mu_{30}^{12 / 11} \dot{M}_{16}^{-4 / 11} v_{8}^{4}\left(\frac{P_{\text {orb }}}{100 \text { days }}\right) \mathrm{s},
$$

where $v_{8}=v /\left(1000 \mathrm{~km} \mathrm{~s}^{-1}\right)$ is the relative velocity between the stellar wind and the NS.

The most uncertain parameter in Equation (6) is $v_{8}$. The wind velocity strongly affects $P_{\text {eq }}$, but it is not well known for the late-type $M$ giants due to lack of observations. Existing measurements for wind speeds are mainly available for massive OB or Wolf-Rayet stars with high mass-loss rates $\left(\dot{M}_{\text {wind }}=\right.$ $\left.10^{-10}-10^{-5} M_{\odot} \mathrm{yr}^{-1}\right)$. These radiatively driven winds are very fast (up to $\sim 2500 \mathrm{~km} \mathrm{~s}^{-1}$; Lamers \& Cassinelli 1999). The situation is different for SyXB systems. Here no strong evidence for fast winds exists. Late F-M stars are usually thought to have smaller mass-loss rates $\left(\lesssim 10^{-10} M_{\odot} \mathrm{yr}^{-1}\right)$ with much slower terminal velocities $\left(\lesssim 100 \mathrm{~km} \mathrm{~s}^{-1}\right.$; Espey \& Crowley 2008). These slower speeds are consistent with the lower escape 


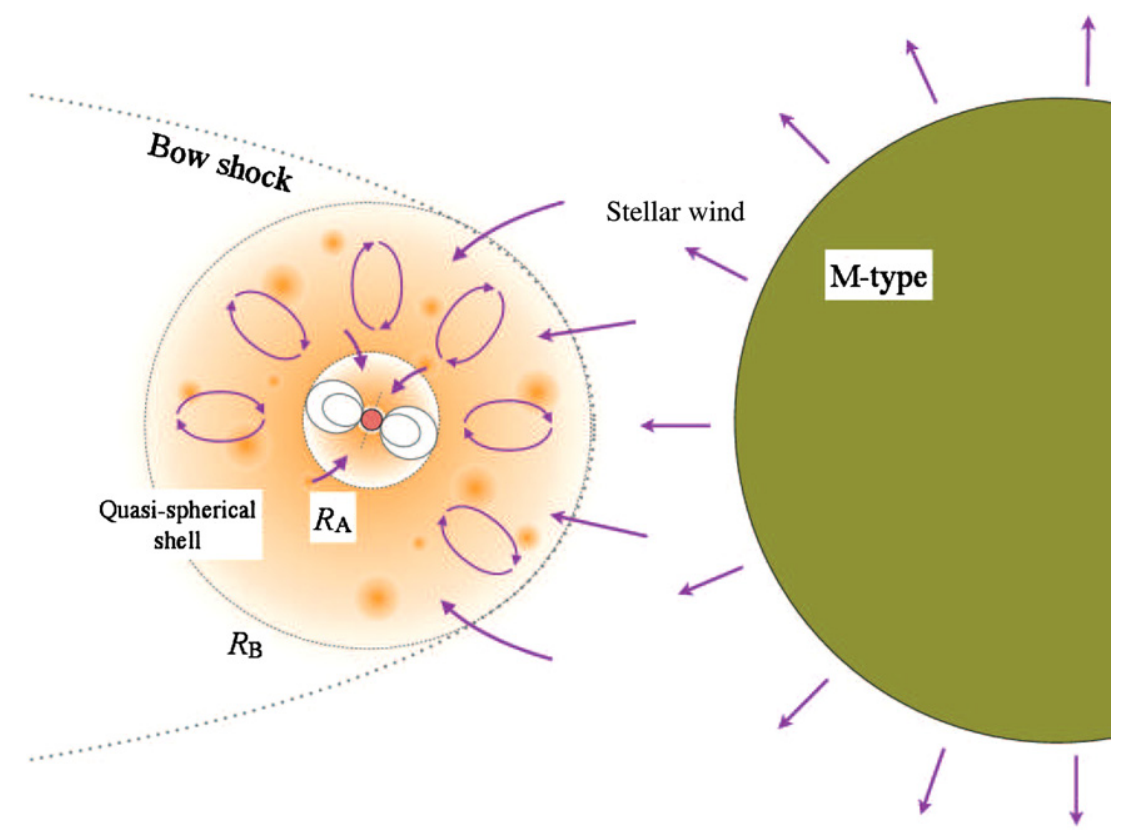

Figure 16. Schematic view of the quasi-spherical accretion and subsonic settling accretion (see details in Shakura et al. 2014). $R_{A}$ and $R_{B}$ are the Alfvén and Bondi radii, respectively.

(A color version of this figure is available in the online journal.)

velocities of the stars. For example, if the donor in 4U 1954+31 has $1.2 M_{\odot}$ and a radius of $80 R_{\odot}$ as suggested by Mattana et al. (2006), then the escape velocity from the companion star would be only $\sim 76 \mathrm{~km} \mathrm{~s}^{-1}$. To our knowledge, the only direct measurement of the wind speed in a SyXB was performed for the M6-giant V2116 Oph, the donor star of the SyXB GX 1+4, where the wind speed was found to be $\sim 100-200 \mathrm{~km} \mathrm{~s}^{-1}$ (Chakrabarty et al. 1998; Hinkle et al. 2006).

Assuming that the wind in $4 \mathrm{U} 1954+319$ is comparable to that in GX 1+4, Figure 17 shows the dependence of the equilibrium period for several plausible different combinations of $v_{8}, P_{\mathrm{orb}}$, $\dot{M}$, and $B$ as calculated with Equation (6). If $4 \mathrm{U} 1954+319$ has a typical accreting NS with a $B$-field of $10^{12-13} \mathrm{G}$, then its equilibrium period can only be explained by a fast $\mathrm{M}$-star wind (e.g., $\sim 300 \mathrm{~km} \mathrm{~s}^{-1}$, probably even faster than that observed in GX 1+4) and the long orbital period (e.g., 500 days).

\subsection{Timescales of Irregular Flares}

A further handle on the accretion mechanism at work in $4 \mathrm{U} 1954+319$ comes from the short-term variability of the source. The quiescent state in 2011 shows a steady weak accretion (in Figures 4(a) and (b)), while the outburst in 2012 exhibits many irregular flare on timescales of $\lesssim 100$ s (Figures 3 and 4 (c) and (d)). This highly variable fluctuation indicates a blobby mass accretion (Figure 16). The X-ray light curves show that the X-ray variation is mainly due to short-timescale spiky flares. Similar spike-like structures, also called "shots," were studied previously in analyses of the light curves of accreting black holes such as Cygnus X-1 (Negoro et al. 1994; Yamada et al. 2013). In these objects, the spectrum gradually softens during the peak of a shot, followed by an immediate hardening. Figure 18 shows a similar analysis for $4 \mathrm{U} 1954+319$. The shots in the light curves are represented by a shape with a nearly symmetric rise and decay (Figures 18(a)-(c)) on a timescale of $\sim 50 \mathrm{~s}$ (at which the count rate drops below the average) with signs of a slight hardening in the early phase of the shot-like behavior which is indicated in (Figures 18(d) and (e)).
A possible explanation for such shots in $4 \mathrm{U} 1954+319$ are individual blobby structures in the accretion flow. To see whether this interpretation is reasonable, we need to take a look at the typical size and timescales of this system (see Figure 17 right panel for a visualization). In the model by Postnov et al. (2012), the stellar wind is gravitationally captured by the NS inside the Bondi radius,

$$
R_{\mathrm{B}}=\frac{2 G M_{\mathrm{NS}}}{v^{2}}=3.78 \times 10^{10} v_{8}^{-2} \mathrm{~cm} .
$$

For $v_{8}=0.3$, i.e., a stellar wind velocity of $300 \mathrm{~km} \mathrm{~s}^{-1}$, the Bondi radius $R_{\mathrm{B}} \sim 4.2 \times 10^{11} \mathrm{~cm}$. Inside this radius a quasistatic shell is formed above the magnetosphere. This is the reservoir of matter from which the NS accretes. In this model the co-rotation radius is (Postnov et al. 2012)

$$
R_{\mathrm{c}}=\left(\frac{G M P^{2}}{4 \pi^{2}}\right)^{1 / 3}=1.21 \times 10^{11} \mathrm{~cm}\left(\frac{P}{5.40 \mathrm{hr}}\right)^{2 / 3} .
$$

Even though the co-rotation radius $R_{\mathrm{c}}$ is very large, it is still inside the Bondi radius if the stellar wind is slower than $\sim 600 \mathrm{~km} \mathrm{~s}^{-1}$ (Figure 17 right). This sets an upper limit for the wind speed in the system.

The size of the magnetosphere, characterized by the Alfvén radius where the gas pressure becomes equal to the magnetic pressure (Ghosh \& Lamb 1979), is modified for quasi-spherical accretion at low mass accretion rates and becomes (Postnov et al. 2012; Shakura et al. 2013)

$$
R_{\mathrm{A}} \sim 1.6 \times 10^{9} \mathrm{~cm}\left(\frac{\mu_{30}^{3}}{\dot{M}_{16}}\right)^{2 / 11} .
$$

With $\dot{M}_{16}=0.38$, we find $R_{\mathrm{A}}=1.9 \times 10^{9} \mathrm{~cm}$ for $B=10^{12} \mathrm{G}$, and $6.7 \times 10^{9} \mathrm{~cm}$ for $B=10^{13} \mathrm{G}$, respectively. This means that the Alfvén radius is smaller by 1-2 orders of magnitudes than $R_{\mathrm{c}}$ and $R_{\mathrm{B}}$. 

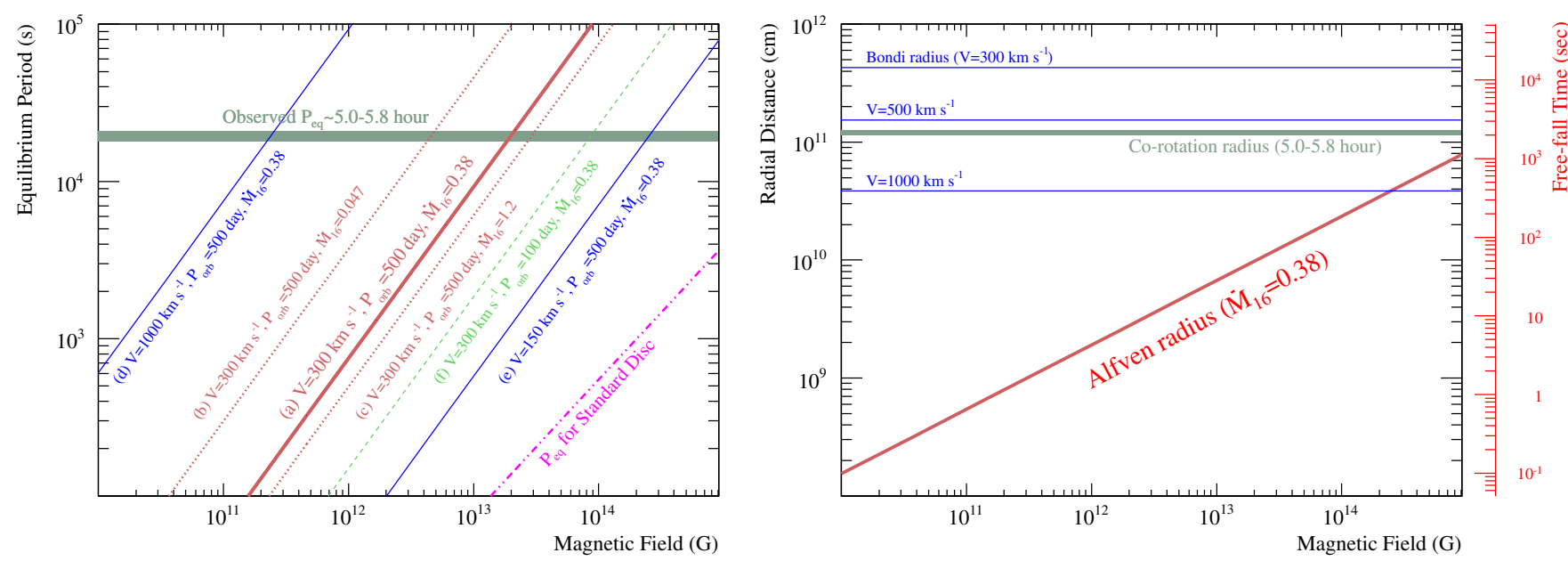

Figure 17. Left: the NS equilibrium period $P_{\mathrm{eq}}$ as a function of the magnetic field $B$ and different stellar wind parameters (velocity, mass accretion rate, and fixed $P_{\text {orb }}$ at 500 days) according to the quasi-spherical accretion model (Postnov et al. 2012, see Equation (6)). The green box in the background shows the observed equilibrium period of $4 \mathrm{U} 1954+319,5.0-5.8 \mathrm{hr}$. The magenta line at high $B$-fields shows the predicted equilibrium period for a standard disk (Equation (5) and Ghosh \& Lamb 1979). Right: the Alfvén radius from Equation (9) as a function of the $B$-field strength and Bondi radii (Equation (7)) for different wind speeds (1000, 500, and $300 \mathrm{~km} \mathrm{~s}^{-1}$ ), assuming the observed $\dot{M}_{16}=1.6$. The green box shows the co-rotation radius. The right hand axis shows the free-fall time onto the neutron star from each radius.

(A color version of this figure is available in the online journal.)

This estimate for the size of the Alfvén radius is roughly consistent with that found from spectral analysis. While the 2012 spectrum is Comptonized, during the 2011 quiescent state Comptonization does not play a strong role. Approximating the quiescent spectrum by a blackbody and using the Stefan-Boltzmann law, the hot spot size on the NS is $R_{\text {hot }}=77 \mathrm{~m} \cdot(d / 1.7 \mathrm{kpc})$. Since the hot spot size is determined by the location where matter couples onto the $B$-field, we can use this measurement to estimate the size of the Alfvén radius (Lamb et al. 1973),

$$
R_{\mathrm{A}} \lesssim 10^{5} \mathrm{~km}\left(\frac{R_{\mathrm{hot}}}{100 \mathrm{~m}}\right)^{-2} \sim 5.9 \times 10^{9} \mathrm{~cm} .
$$

The measured hot spot radius thus yields a $B$-field in the $10^{12}-10^{13} \mathrm{G}$ range (Figure 17).

Depending on the ability of the plasma to enter the magnetosphere, matter from the magnetosphere funnels onto the NS poles along the $B$-field lines. The free-fall time is given by

$$
t_{\mathrm{ff}}=51 \mathrm{~s}\left(\frac{r}{10^{10} \mathrm{~cm}}\right)^{3 / 2} \text {. }
$$

The free-fall timescale from the Alfvén radius for $B \sim 10^{13} \mathrm{G}$ is $\sim 30 \mathrm{~s}$, close to the typical timescale of individual shots (Figure 18). Thus, it is possible to interpret the shot-like flares as produced during the free-fall of the blobs from the shell above the magnetosphere. Even though the cooling timescale of the matter is complicated when considering different cooling processes, if we assume Compton cooling as an effective process (Shakura et al. 2012), the cooling time,

$$
t_{\text {cool }}=10^{3} \mathrm{~s} \cdot \frac{1}{\dot{M}_{16}}\left(\frac{r}{10^{10} \mathrm{~cm}}\right)^{2}
$$

becomes $\sim 1200 \mathrm{~s}$ at $B=10^{13} \mathrm{G}$ and $\dot{M}_{16}=0.38$. This is longer than the typical free-fall time $\left(t_{\text {cool }}>t_{\mathrm{ff}}\right)$, which satisfies the assumption of a low-luminosity quasi-spherical accretion.

The log-normal distribution of the X-ray light curve count rates (Figure 11) is also consistent with the picture of quasispherical magnetospheric accretion. As suggested by Fürst et al. (2010), such a log-normal distribution implies that the underlying physical process should be multiplicative rather than additive, since addition of many independent emissions will produce a Gaussian distribution due to the central limit theorem. Such a process could be induced via a positive feedback loop, where blob accretion induces subsequent accretion, e.g., via enhanced Compton cooling, potentially creating positive feedback until the matter at the boundary of the magnetosphere is exhausted. Alternatively, the structures leading to a lognormal distribution of the accretion rate could also be due to a highly inhomogeneous and clumpy stellar wind from the $\mathrm{M}$-giant companion or due to turbulent processes in the spherical shell itself.

\subsection{Absorption in the Spherical Shell}

Finally, let us compare the above accretion scheme with the absorption properties of the circumstellar material. These come from both the absorption modeling of the X-ray continuum and the analysis of the narrow $\mathrm{Fe}-\mathrm{K} \alpha$ line at $6.4 \mathrm{keV}$. The latter was detected with Suzaku in the 2012 observation and has been previously reported with BeppoSAX $(\mathrm{EW}=51 \pm 20 \mathrm{eV})$ and RXTE $\left(48_{-10}^{+13} \mathrm{eV}\right.$; Masetti et al. 2007b). A narrow iron line feature is characteristic to HMXB-NSs rather than LMXB-NSs.

As a plausibility check, we assume that $N_{\mathrm{H}}$ is directly related to the properties of the shell in the low-luminosity wind accretion model. Using the Suzaku-derived $N_{\mathrm{H}}$, the number density $\left(n_{\mathrm{H}}\right)$ inside the spherical shell $\left(R<R_{\mathrm{B}}\right)$ becomes $\left\langle n_{\mathrm{H}}\right\rangle=N_{\mathrm{H}} / R_{\mathrm{B}}=$ $1.9 \times 10^{10} \mathrm{~cm}^{-3}\left(v_{8}=0.3\right)$, corresponding to a mass density of $\langle\rho\rangle=\left\langle n_{\mathrm{H}}\right\rangle m_{\mathrm{p}} \sim 3.2 \times 10^{-14} \mathrm{~g} \mathrm{~cm}^{-3}$, where $m_{\mathrm{p}}$ is the proton mass. If the NS is fed by blobs of matter which have the density of the shell at the Alfén radius, then in order to reach the average mass accretion rate $\left\langle\dot{M}_{16}\right\rangle=4 / 3 \pi R_{\text {blob }}^{3} \rho / t_{\text {shot }}$, where $R_{\text {blob }}$ and $t_{\text {shot }}$ are the typical blob size and shot timescale, we find $R_{\text {blob }} \sim 8.0 \times 10^{9} \mathrm{~cm}$ with $\left\langle\dot{M}_{16}\right\rangle=0.38$ and $t_{\text {shot }} \sim 50 \mathrm{~s}$. It is intriguing that this initial blob radius is comparable to the Alfvén radius (e.g., $R_{\mathrm{A}}=6.8 \times 10^{9} \mathrm{~cm}$ at $B=10^{13} \mathrm{G}$ ) and is much smaller than the quasi-spherical shell size $R_{\mathrm{B}}$. Thus, the shell can store enough mass to generate the observed multiple flares and to sustain accretion for longer time intervals. 


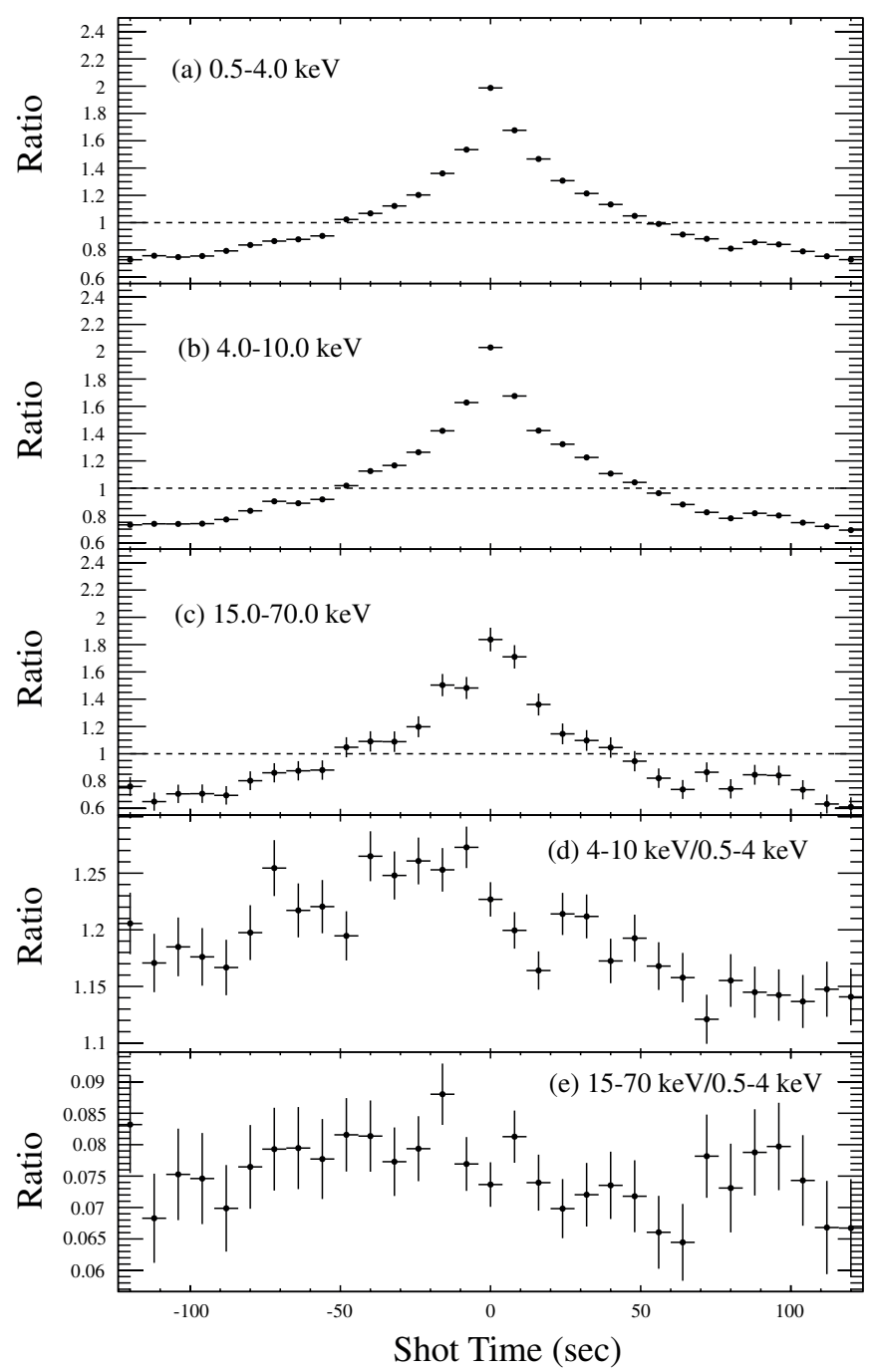

Figure 18. Background-subtracted stacked shot profiles of $4 \mathrm{U} 1954+31$ in (a) the 0.5-4.0 keV, (b) 4.0-10.0 keV, and (c) 15.0-70.0 keV bands. Shot peaks were determined from local maxima within $\pm 200 \mathrm{~s}$ duration and with a threshold factor of $f=1.8$ (Yamada et al. 2013). The profiles in panels (a)-(c) are normalized by the average rate in individual energy bands. Panels (d) and (e) show the divisions of the profiles in panels (b) and (c) by that in panel (a).

Finally, as a cross check, we note that the ionization parameter of the shell at the Bondi radius is $\xi=L_{\mathrm{X}} /\left\langle n_{\mathrm{H}}\right\rangle r^{2}=2.1 \times$ $10^{4}\left(r / 10^{10} \mathrm{~cm}\right)^{-2} \sim 11$, i.e., the outer part of the shell is at most moderately ionized, making it possible to produce the $6.4 \mathrm{keV}$ line in the shell.

\section{CONCLUSION}

We analyzed two Suzaku data sets, obtained in 2011 and 2012, of the SyXB 4U 1954+319 consisting of the slowly rotating 5.4 hr X-ray pulsar and a late type primary star (M4-5 III). Our main results are as follows.

1. We reconfirmed the slow rotation period and the large period fluctuations. We also identified several additional features of $4 U$ 1954+319, including a hard continuum without a clear CRSF, a narrow $\mathrm{Fe}-\mathrm{K}_{\alpha}$ line, and sporadic flares. The continuum was harder when the source was brighter.

2. From spectral and timing properties, $4 \mathrm{U} 1954+319$ is suggested to have a strong $B$-field of $\gtrsim 10^{12-13} \mathrm{G}$. This is supported by the high PF of $60 \%-80 \%$, the slow rotation, the hard featureless continuum, and the narrow $\mathrm{Fe}-\mathrm{K} \alpha$ line.

3. The NS is expected to be close to equilibrium because of the four spin transitions and large period fluctuations. Such an evolution can be explained by a quasi-spherical accretion in a subsonic accretion regime which is considered to apply to $4 \mathrm{U} 1954+319$ because of its low luminosity $\left(0.023-2.1 \times 10^{35} \mathrm{erg} \mathrm{s}^{-1}\right)$, presumably wide orbital period, and rather low wind velocity from the M-type giant.

4. Recurrent irregular flares during the outburst have a typical timescale of $\sim 50 \mathrm{~s}$, which is interpreted as intermittent accretion from the Alfvén radius of the $\sim 10^{13} \mathrm{G}$ NS. The log-normal distribution of the light curve indicates an underlying multiplicative process (e.g., positive feedback) in the accretion.

5. The extreme magnetar-like extreme $B$-field $\left(10^{16} \mathrm{G}\right)$ derived from the standard disk accretion in Equation (5) is not required if we assume a low luminosity and quasi-spherical accretion.

6. The presence of a system like $4 \mathrm{U} 1954+319$, which is very likely to involve a high-field NS, provides another example that urges us to challenge the conventional classification of NS binaries into LMXBs and HMXBs.

The authors would like to express their thanks to the Suzaku team for their prompt observation during the 2012 flaring activity. T.E. was supported by JSPS KAKENHI, Grant-inAid for JSPS Fellows, 24-3320. We thank Pranab Ghosh and Hiromitsu Takahashi for useful discussions on this source, Kunugawa Tomoya and Kenta Hotokezaka for comments on the binary evolution. We thank the Deutsches Zentrum für Luftund Raumfahrt for partial funding under DLR grant number 50 OR 1207.

\section{REFERENCES}

Asplund, M., Grevesse, N., Sauval, A. J., \& Scott, P. 2009, ARA\&A, 47, 481 Balucinska-Church, M., \& McCammon, D. 1992, ApJ, 400, 699 Bodaghee, A., Courvoisier, T. J.-L., Rodriguez, J., et al. 2007, A\&A, 467, 585 Bodaghee, A., Rahoui, F., Tomsick, J. A., \& Rodriguez, J. 2012, ApJ, 751, 113 Bozzo, E., Falanga, M., \& Stella, L. 2008, ApJ, 683, 1031

Caballero, I., \& Wilms, J. 2012, MmSAI, 83, 230

Camero-Arranz, A., Pottschmidt, K., Finger, M. H., et al. 2012, A\&A, 546, A40 Chakrabarty, D., \& Roche, P. 1997, ApJ, 489, 254

Chakrabarty, D., van Kerkwijk, M. H., \& Larkin, J. E. 1998, ApJL, 497, L39

Corbet, R., Barbier, L., Barthelmy, S., et al. 2006, ATeL, 797

Corbet, R. H. D. 1986, MNRAS, 220, 1047

Corbet, R. H. D., Sokoloski, J. L., Mukai, K., Markwardt, C. B., \& Tueller, J. 2008, ApJ, 675, 1424

Davidsen, A., Malina, R., \& Bowyer, S. 1977, ApJ, 211, 866

Enoto, T., Makishima, K., Terada, Y., et al. 2008, PASJ, 60, 57

Enoto, T., Nakazawa, K., Makishima, K., et al. 2010a, PASJ, 62, 475

Enoto, T., Nakazawa, K., Makishima, K., et al. 2010b, ApJL, 722, L162

Enoto, T., Nakagawa, Y. E., Rea, N., et al. 2009, ApJL, 693, L122

Espey, B. R., \& Crowley, C. 2008, in ASP Conf. Ser. 401, RS Ophiuchi (2006) and the Recurrent Nova Phenomenon, ed. A. Evans, M. F. Bode, T. J. O'Brien, \& M. J. Darnley (San Francisco, CA: ASP), 166

Farrell, S. A., Sood, R. K., O’Neill, P. M., \& Dieters, S. 2008, MNRAS, 389, 608

Finley, J. P., Belloni, T., \& Cassinelli, J. P. 1992, A\&A, 262, L25

Forman, W., Jones, C., Cominsky, L., et al. 1978, ApJS, 38, 357

Fukazawa, Y., Mizuno, T., Watanabe, S., et al. 2009, PASJ, 61, 17

Fürst, F., Kreykenbohm, I., Pottschmidt, K., et al. 2010, A\&A, 519, A37

Fürst, F., Kreykenbohm, I., Suchy, S., et al. 2011a, A\&A, 525, A73

Fürst, F., Marcu, D. M., Pottschmidt, K., et al. 2011b, arXiv:1106.2708

Garcia, M., Baliunas, S. L., Elvis, M., et al. 1983, ApJ, 267, 291

Ghosh, P., \& Lamb, F. K. 1979, ApJ, 232, 259

González-Galán, A., Kuulkers, E., Kretschmar, P., et al. 2012, A\&A, 537, A66 
Grevesse, N., \& Anders, E. 1989, in AIP Conf. Proc. 183, Cosmic Abundances of Matter, ed. C. J. Waddington (Melville, NY: AIP), 1

Hinkle, K. H., Fekel, F. C., Joyce, R. R., et al. 2006, ApJ, 641, 479

Ho, W. C. G., Klus, H., Coe, M. J., \& Andersson, N. 2014, MNRAS, 437, 3664

Ikhsanov, N. R. 2007, MNRAS, 375, 698

Inoue, H. 1985, SSRv, 40, 317

Ishisaki, Y., Maeda, Y., Fujimoto, R., et al. 2007, PASJ, 59, 113

Kalberla, P. M. W., Burton, W. B., Hartmann, D., et al. 2005, A\&A, 440, 775

Kaplan, D. L., Levine, A. M., Chakrabarty, D., et al. 2007, ApJ, 661, 437

Kitamura, Y., Takahashi, H., \& Fukazawa, Y. 2014, PASJ, 66, 6

Klus, H., Ho, W. C. G., Coe, M. J., Corbet, R. H. D., \& Townsend, L. J. 2014, MNRAS, 437, 3863

Kouveliotou, C., Dieters, S., \& Strohmeyer, T. 1998, Natur, 393, 253

Koyama, K., Tsunemi, H., Dotani, T., et al. 2007, PASJ, 59, 23

Lamb, F. K., Pethick, C. J., \& Pines, D. 1973, ApJ, 184, 271

Lamers, H. J. G. L. M., \& Cassinelli, J. P. (ed.) 1999, Introduction to Stellar Winds (Cambridge: Cambridge Univ. Press), 452

Leahy, D. A., Elsner, R. F., \& Weisskopf, M. C. 1983, ApJ, 272, 256

Li, X.-D., \& van den Heuvel, E. P. J. 1999, ApJL, 513, L45

Liu, Q. Z., van Paradijs, J., \& van den Heuvel, E. P. J. 2006, A\&A, 455, 1165

Liu, Q. Z., van Paradijs, J., \& van den Heuvel, E. P. J. 2007, A\&A, 469, 807

Lü, G.-L., Zhu, C.-H., Postnov, K. A., et al. 2012, MNRAS, 424, 2265

Lutovinov, A., \& Tsygankov, S. 2008, in AIP Conf. Ser. 1054, Cool Discs, Hot Flows: The Varying Faces of Accreting Compact Objects, ed. M. Axelsson (Melville, NY: AIP), 191

Makishima, K., Mihara, T., Nagase, F., \& Tanaka, Y. 1999, ApJ, 525, 978

Makishima, K., Ohashi, T., Sakao, T., et al. 1988, Natur, 333, 746

Marcu, D. M., Fürst, F., Pottschmidt, K., et al. 2011, ApJL, 742, L11

Masetti, N., Dal Fiume, D., Cusumano, G., et al. 2002, A\&A, 382, 104

Masetti, N., Landi, R., Pretorius, M. L., et al. 2007a, A\&A, 470, 331

Masetti, N., Nucita, A. A., \& Parisi, P. 2012, A\&A, 544, A114

Masetti, N., Orlandini, M., dal Fiume, D., et al. 2006a, A\&A, 445, 653

Masetti, N., Orlandini, M., Palazzi, E., Amati, L., \& Frontera, F. 2006b, A\&A, 453, 295

Masetti, N., Rigon, E., Maiorano, E., et al. 2007b, A\&A, 464, 277

Mattana, F., Götz, D., Falanga, M., et al. 2006, A\&A, 460, L1

Mihara, T. 1995, PhD thesis, Univ. Tokyo

Mitsuda, K., Bautz, M., Inoue, H., et al. 2007, PASJ, 59, 1

Moretti, A., Pagani, C., Cusumano, G., et al. 2009, A\&A, 493, 501

Nagae, O., Takahashi, H., Shirai, H., \& Fukazawa, Y. 2008, Proc. VII Microquasar Workshop: Microquasars and Beyond, PoS(MQW7) (Trieste: SISSA), 102

Negoro, H., Miyamoto, S., \& Kitamoto, S. 1994, ApJL, 423, L127

Nespoli, E., Fabregat, J., \& Mennickent, R. E. 2010, A\&A, 516, A94

Nomoto, K., \& Kondo, Y. 1991, ApJL, 367, L19
Patel, S. K., Zurita, J., Del Santo, M., et al. 2007, ApJ, 657, 994

Patruno, A., \& Watts, A. L. 2012, arXiv:1206.2727

Pearlman, A. B., Corbet, R. H. D., Pottschmidt, K., \& Skinner, G. K. 2011 , AAS/High Energy Astrophysics Division, 12, \#42.06

Perna, R., Bozzo, E., \& Stella, L. 2006, ApJ, 639, 363

Postnov, K., Shakura, N., González-Galán, A., et al. 2010, in Proc. 8th INTEGRAL Workshop, The Restless Gamma-ray Universe (Trieste, Italy: Proceedings of Science), 15

Postnov, K., Shakura, N. I., Kochetkova, A. Y., \& Hjalmarsdotter, L. 2012, in Proc. 9th INTEGRAL Workshop, An INTEGRAL View of the High-energy Sky (Trieste, Italy: Proceedings of Science), 22

Reig, P., Torrejón, J. M., \& Blay, P. 2012, MNRAS, 425, 595

Reig, P., Torrejón, J. M., Negueruela, I., et al. 2009, A\&A, 494, 1073

Sakurai, S., Yamada, S., Torii, S., et al. 2012, PASJ, 64, 72

Sasano, M., Makishima, K., Sakurai, S., Zhang, Z., \& Enoto, T. 2013, arXiv: 1311.4618

Schönherr, G., Wilms, J., Kretschmar, P., et al. 2007, A\&A, 472, 353

Serlemitsos, P. J., Soong, Y., Chan, K.-W., et al. 2007, PASJ, 59, 9

Shakura, N., Postnov, K., Kochetkova, A., \& Hjalmarsdotter, L. 2012, MNRAS, 420, 216

Shakura, N. I., Postnov, K. A., Kochetkova, A. Y., \& Hjalmarsdotter, L. 2013, PhyU, 56, 321

Shakura, N. I., Postnov, K. A., Kochetkova, A. Y., \& Hjalmarsdotter, L. 2014, in European Physical Journal Web of Conferences, Physics at the Magnetospheric Boundary, ed. E. Bozzo et al. (Les Ulis, France: EDP Sciences), 2001

Smith, R. K., Brickhouse, N. S., Liedahl, D. A., \& Raymond, J. C. 2001, ApJL, 556, L91

Sugizaki, M., Mihara, T., Nakagawa, Y. E., et al. 2010, ATel, 2702, 1

Takahashi, T., Abe, K., Endo, M., et al. 2007, PASJ, 59, 35

Thompson, C., \& Duncan, R. C. 1995, in ASP Conf. Ser. 72, Millisecond Pulsars: A Decade of Surprise, ed. A. S. Fruchter, M. Tavani, \& D. C. Backer (San Francisco, CA: ASP), 301

Titarchuk, L., \& Lyubarskij, Y. 1995, ApJ, 450, 876

Torrejón, J. M., Schulz, N. S., Nowak, M. A., \& Kallman, T. R. 2010, ApJ, 715,947

Tweedy, R. W., Warwick, R. S., \& Remillard, R. 1989, Two Topics in X-Ray Astronomy, Volume 1: X Ray Binaries. Volume 2: AGN and the X Ray Background (Noordwijk: ESA), 661

Verner, D. A., Ferland, G. J., Korista, K. T., \& Yakovlev, D. G. 1996, ApJ, 465,487

Warwick, R. S., Marshall, N., Fraser, G. W., et al. 1981, MNRAS, 197,865

Wilms, J., Allen, A., \& McCray, R. 2000, ApJ, 542, 914

Yamada, S., Negoro, H., Torii, S., et al. 2013, ApJL, 767, L34 